Article

\title{
Buellia dispersa (Lichens) Used as Bio-Indicators for Air Pollution Transport: A Case Study within the Las Vegas Valley, Nevada (USA)
}

\author{
Douglas B. Sims ${ }^{1, *}$ (D), Amanda C. Hudson ${ }^{1}$, Ji Hye Park ${ }^{2}$, Vernon Hodge ${ }^{2}$, Heidi Porter ${ }^{3}$ and \\ W. Geoffrey Spaulding ${ }^{4}$ \\ 1 Department of Physical Sciences, College of Southern Nevada, North Las Vegas, NV 89030, USA; \\ amanda.hudson@csn.edu \\ 2 Department of Chemistry, University of Nevada Las Vegas, Las Vegas, NV 89154, USA; \\ parkj37@unlv.nevada.edu (J.H.P.); hodgev@unlv.nevada.edu (V.H.) \\ 3 Department of Biological Sciences, College of Southern Nevada, North Las Vegas, NV 89030, USA; \\ heidi.Porter@csn.edu \\ 4 Terra Antiqua Research, Henderson, NV 89074, USA; wgeoffrey@terrantiqua.com \\ * Correspondence: douglas.sims@csn.edu; Tel.: +1-512-809-5094
}

Received: 7 November 2017; Accepted: 13 December 2017; Published: 17 December 2017

\begin{abstract}
Hazardous substances (e.g., toxic elements, oxides of nitrogen, carbon and sulfur) are discharged to the environment by a number of natural and anthropogenic activities. Anthropogenic air pollution commonly contains trace elements derived from contaminants and additives released into the atmosphere during fossil fuel combustion (automobiles, power generation, etc.) as well as physical processes (e.g., metal refining, vehicle brake wear, and tire and pavement wear). Analysis of pollutant chemical concentrations in lichens collected across the Las Vegas Valley allows documentation of the distribution of air pollution in the Valley. Analyses of lichen biomass (Buellia dispersa), when compared to windrose diagrams, shows pathways of airborne pollutant transport across the Las Vegas Valley. The west and north sectors of the Las Vegas Valley contained the lowest target contaminates (e.g., $\mathrm{Cr}, \mathrm{Cu}, \mathrm{Co}, \mathrm{Pb}, \mathrm{Ni}$ ) and the highest $\mathrm{NO}_{3}{ }^{-}$while the east and south sectors contained the highest levels of target contaminates and lowest $\mathrm{NO}_{3}{ }^{-}$. Additionally, metals and $\mathrm{NO}_{3}{ }^{-}$detected in the east and south sectors of the valley indicate that air pollution generated in the valley is moving from the south to the north-northeast and across the valley, exiting on the north and south side of Frenchman Mountain.
\end{abstract}

Keywords: Las Vegas; Buellia dispersa; lichen; air pollution; trace metals; nitrates; windrose

\section{Introduction}

The United States Agency for Toxic Substances and Disease Registry (USATSDR) ranks certain metals (e.g., $\mathrm{As}, \mathrm{Pb}, \mathrm{Cd}, \mathrm{Cr}$ ) as priority pollutants for known health effects [1]. Polluted air can typically contain $\mathrm{NO}_{\mathrm{x}}, \mathrm{SO}_{\mathrm{x}}, \mathrm{CO}_{2}$, aerosols, particulate matter $\left(\mathrm{PM}_{10}, \mathrm{PM}_{2.5}\right)$, heavy ("trace") metals, and organic pollutants from sources of fossil fuel activities [2,3]. Some metals (e.g., $\mathrm{Pb}, \mathrm{Cd}, \mathrm{Cr}$ ) are known to be hazardous because of bioaccumulation and long retention times in tissues of living organisms, including humans [2]. These pollutants are not only suspended in air as particulates, but are found in tissues of plant and animal species within a given area, resulting from deposition onto and uptake processes. Studies have characterized the magnitude and impact of pollution transport over great distances as challenging to control from regulatory and public health perspectives $[4,5]$.

Researchers have linked air pollution to health effects such as upper respiratory problems, asthma, lung disease, resulting in an increase to healthcare costs [6]. Studies have shown that pollution 
detected in a particular city can be a mixture of both local and external population centers [3,6-8]. With this understanding, monitoring the distribution and depositional patterns of pollutants are of great importance.

Researchers have indicated that lichens in populated areas are on a decline due to anthropogenic atmospheric pollution [9]. Authors have shown that lichens are useful bio-indicators of air pollution and have been utilized across the United States and Europe [10-12]. Developed areas, especially those located in geographic basins, have been shown to be more prone to air pollution (e.g., trace elements ("metals"), $\mathrm{CO}, \mathrm{CO}_{2}, \mathrm{NO}_{\mathrm{x}}$, and $\mathrm{SO}_{\mathrm{x}}$ ) in winter months as a result of various atmospheric and weather related processes [13,14]. Giordano et al. [15] utilized lichen species in Naples, Italy for biomonitoring, owing to its physical nature (i.e., lack of roots, high surface area to volume ratio, slow growing rate $(<1 \mathrm{~mm} /$ year), and lack of cuticles. Additionally, it has a thin layer of dead tissue preventing pathogens from infecting the matrix area [13]. Jeran et al. [16] and others also used lichens for monitoring air pollution because of their high accumulation of metals compared to its physiological requirements, dormancy during dry or drought conditions, slow growth rate, and their retention and preservation of atmospheric pollutants over long periods [17-20].

Evaluating air pollution in the Las Vegas Valley has been a focus of the United State Environmental Protection Agency (USEPA) and the Clark County Department of Air Quality; however, evaluating lichen biomass for metals and other pollutants in relation to wind patterns in the Las Vegas Valley has never been included in their studies. The Las Vegas Valley is located in Clark County, Nevada, and is situated in a geographic basin. Since the early 1990s, the population has grown from 500,000 to over two million residents (Figure 1) resulting in air pollution becoming more of an issue. Monitoring the spatial and temporal trends of pollution transport across a vast area can be difficult to characterize. Knowing where and how pollution in the valley is generated and how it migrates is important to the health and wellbeing of residents and visitors. The specific objectives of this study were to:

(1) Identify and quantify trace element uptake by lichen biomass.

(2) Identify and quantify $\mathrm{NO}_{3}{ }^{-}$in lichen biomass.

(3) Assess patterns for metals and $\mathrm{NO}_{3}{ }^{-}$concentrations across the Las Vegas Valley.

(4) Compare spacial distribution of pollutants with wind patterns with respect to metal and $\mathrm{NO}_{3}{ }^{-}$ levels detected in lichen biomass across the valley.

Objectives of this study were to identify transport related patterns for metals and $\mathrm{NO}_{3}{ }^{-}$ enrichment (pollution) within the Las Vegas valley. Based on a field investigation of 72 separate lichen sample locations, it was possible to identify pollution related trends along the outer edge of the valley, distribution of pollution, and possibly external transport of pollution from different areas. This was accomplished with analytical procedures, statistical analysis, and windrose pattern data with regard to spatial relations between four sectors of the Las Vegas valley and population. 


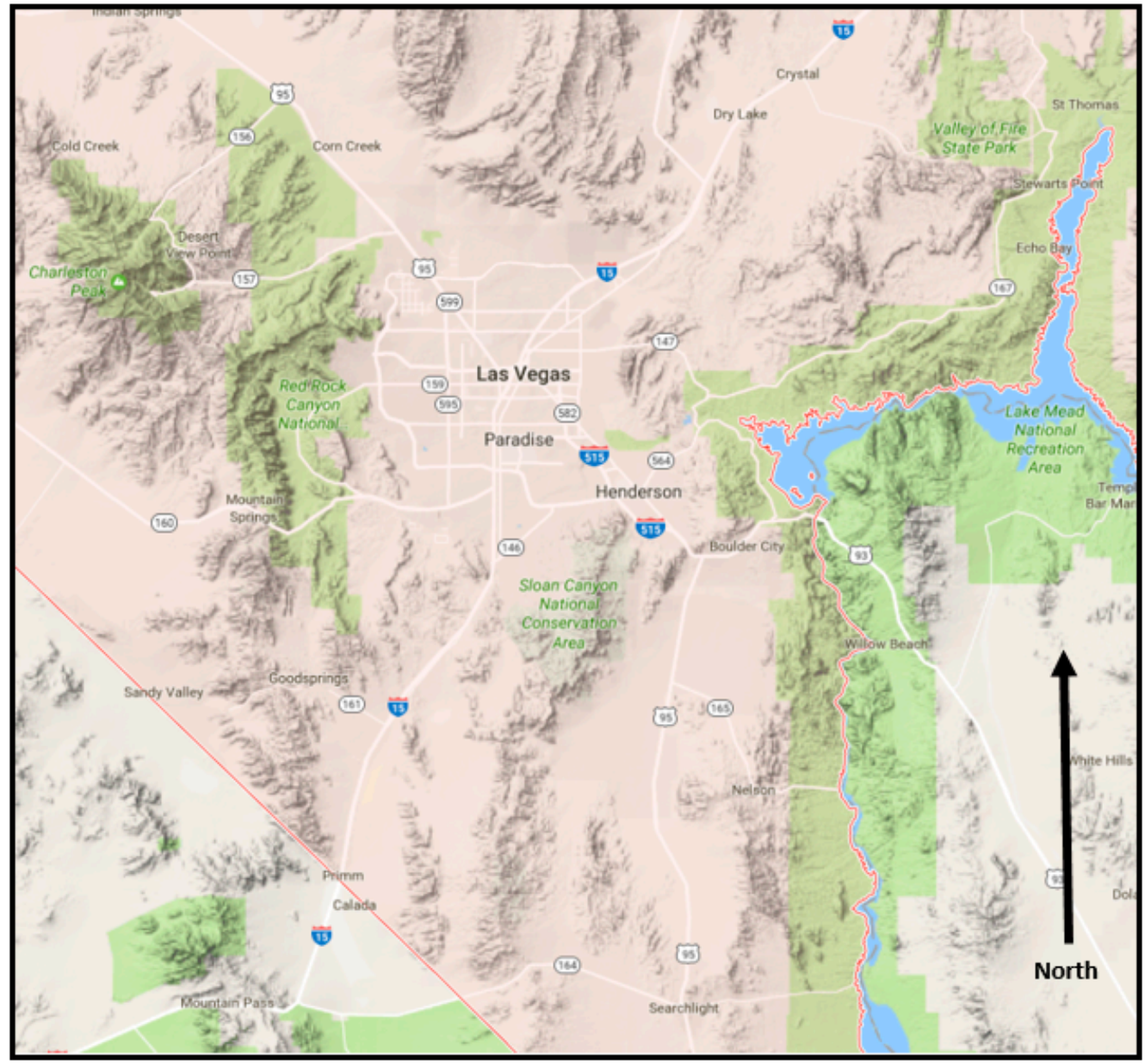

Figure 1. Southern Nevada and the Las Vegas Valley showing north-south trending mountain ranges (Google maps).

\section{Environmental Setting}

\subsection{Study Area}

The study area is located on the development edge of the greater City of Las Vegas within the central Basin and Range. Briefly, mountain ranges in southern Nevada are oriented north-south, likely formed during the Oligocene and Miocene Epochs [21-23]. The area includes the Spring Mountains to the west, Las Vegas and Sheep ranges to the north, Sunrise, Frenchman and River mountains to the east, and the McCullough range to the North and South [24]. Interstate 15 (I-15) enters the valley running from southern California to Las Vegas traverses between the McCullough range North and South and the Spring Mountains (Figure 1). Finally, the Las Vegas Valley is situated within a basin (Las Vegas Basin) ranging between $\sim 610 \mathrm{~m}$ above mean sea level (amsl) along the Las Vegas Wash (east-northeastern part of the valley) to over $3350 \mathrm{~m}$ amsl (Mount Charleston, Spring Mountains).

\subsection{Lichen Species}

Lichens are commonly found across different ecosystems from tundra to subtropical thorn forests with their structure of the thallus varies in part with their habitat and substrate [25]. Ascendant-foliose, hair-like and arborescent lichens are commonly anchored on tree bark and rocks in environments with higher humidity [25]. Lichens are particularly important in foggy communities of montane and coastal habitats however, crustose and squamose lichens with flattened, essentially two-dimensional thalli can be abundant in certain desert and semi-desert habitats. Frequent colonizers of stable rock surfaces, 
particularly igneous rocks, lichens can vary widely in color, depending on the relative amounts of pigments produced.

Crustose and squamose lichen colonies are typical in the southwest desert region owing to a simple near-two dimensional structure that helps control moisture loss in these hyper-arid microhabitats. Researchers have observed petrophytic (rock-loving) lichen colonies persisting for decades on volcanic rocks in the Mojave Desert, particularly in more mesic habitats such as on north-facing slopes or in canyons [25]. Lichens possess roughened, irregular surfaces that are effective in capturing and retaining particulate fall-out [16]. Because of their longevity and slow growth, a particular lichen colony can be resampled for years or decades.

By way of macroscopic identification, the target lichen used for this study is the gray Buellia dispersa sp., a crustose areolate lichen ("Buellia sp.") common on igneous rocks throughout the region though rarely on soils [25]. The growth rates of Buellia sp. vary widely based on substrate, temperature, and moisture levels; however, studies have shown that the radius of colonies expand $\sim 1 \mathrm{~mm} /$ year in desert environments $[19,25]$. The Buellia sp. goes dormant during drought conditions and therefore might have some years when there is little to no detectable growth [25].

\section{Materials and Methods}

\subsection{Sampling Design and Laboratory Procedures}

Due to the large geographic area of this project, sampling was performed between November of 2013 and January of 2014 and again between November 2014 and January 2015 on lands surrounding the Las Vegas Valley. The strategy was to assess concentrations of pollutants (trace elements and $\mathrm{NO}_{3}{ }^{-}$) contained in lichen biomass along the outer edge of the valley to evaluate the distribution of pollution across the Las Vegas Valley. Sixty-six (66) samples were collected from within the Las Vegas Valley and six samples were collected from outside the valley to evaluate background concentrations (Figure 2). Sampling involved the removal of a $10 \times 10 \mathrm{~cm}$ square area containing Buellia dispersa, a crustose areolate lichen ("Buellia sp."), with a plastic scrapper, cleaned between uses, bagged, labeled and placed into a cooler at $4{ }^{\circ} \mathrm{C}$ for transportation to the laboratory. Each sampling location was a single bulk area of $10 \times 10 \mathrm{~cm}$ designed to measure pollutant concentration at that specific location.

Samples were processed according to the United States Environmental Protection Agency's (USEPA) Solid Waste 846 (SW-846) protocols, including quality control measures [26]. Prior to metals ( $\mathrm{Al}, \mathrm{As}, \mathrm{Ba}, \mathrm{Cd}, \mathrm{Cr}, \mathrm{Co} ., \mathrm{Cu}, \mathrm{Mo}, \mathrm{Ni}, \mathrm{Pb}, \mathrm{V}$ and $\mathrm{Zn}$ ) and $\mathrm{NO}_{3}{ }^{-}$extraction, each sample was manually cleaned under a $4 \times$ power microscope to remove sediment and debris from the lichen biomass.

Metals were extracted by United States Environmental Protection Agency (USEPA) Method 3050B (rigorous acid digestion) followed by a Perkin Elmer Optima 3300 Dual View inductively coupled plasma-optical emission spectrometry (ICP-OES) instrument for analysis using USEPA Method 6010B on a Perkin Elmer 3300 Dual-View ICP-OES with the axial view chosen for analysis of samples [26]. Instrument calibration was composed of six external calibration points, including a blank, and fitted by linear regression greater than $0.995^{\mathrm{R}}$ [26]. Analytical integrity was verified with a USEPA certified solid reference sample purchased from RTC Corporation (CRM022-020, Sample 5, lot D522) used to measure the acid digestion procedure. Finally, each sample was processed in triplicate to evaluate reproducibility. Lichens absorb nitrogen from the air in the form of nitrate and ammonium; however, for this study, $\mathrm{N}_{\text {as }} \mathrm{NO}_{3}{ }^{-}$was measured [14,27]. Nitrate $\left(\mathrm{N}\right.$ as $\left.\mathrm{NO}_{3}{ }^{-}\right)$was analyzed per USEPA Method 9210A (Thermo Scientific ${ }^{\mathrm{TM}}$ Orion ${ }^{\mathrm{TM}}$ Nitrate ion selective electrodes: ISE) following SW-846 protocols. 


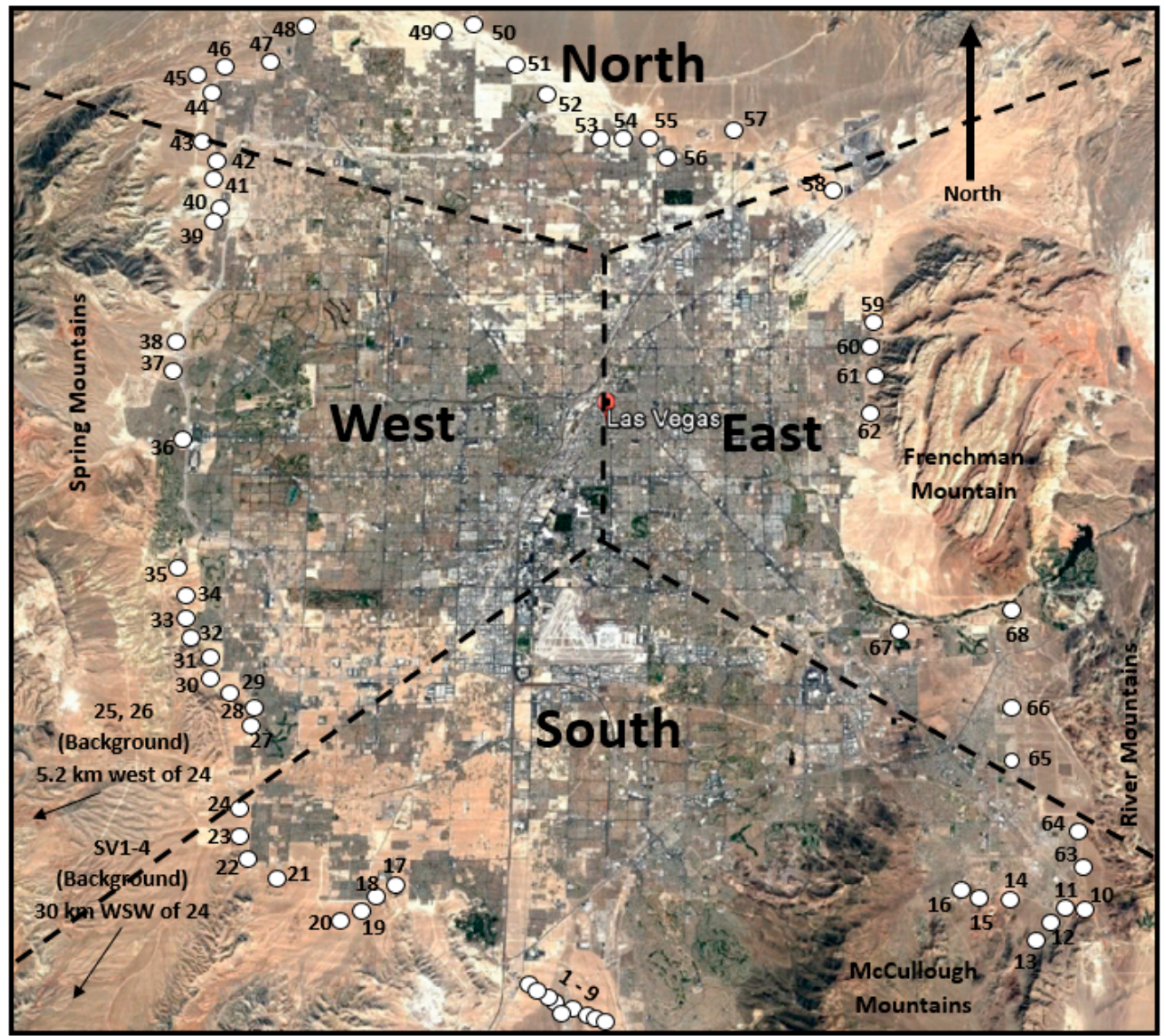

Figure 2. Sample locations and sectors across the Las Vegas Valley (Google maps).

Solubilized nitrate was determined potentiometrically utilizing a nitrate ISE in conjunction with a double-junction reference electrode using a meter equipped with an expanded millivolt scale (mV). First, a portion of each lichen $(\sim 1 \mathrm{~g})$ was measured into a clean, disposable bottle, brought to volume $\left(50 \mathrm{~mL}\right.$ ) with $95^{\circ} \mathrm{C}$ deionized water (DI), capped, agitated for two hours and allowed to cool to room temperature. After agitation, samples were allowed to settle then filtered through a Whatman $42^{\circledR}$ filter paper and measured immediately for $\mathrm{NO}_{3}{ }^{-}$by ion-selective electrode (ISE). During the extraction procedure, bottles were capped immediately after the sample was measured into the bottle, DI water added, agitated, filtered and measured for $\mathrm{NO}_{3}{ }^{-}$immediately by the ISE after filtering to reduce erroneous measurements due to $\mathrm{NO}_{3}{ }^{-}$uptake during the procedure. Finally, a linear calibration was obtained over a range of 5.0 to $100 \mathrm{mg} \mathrm{L}^{-1}$ from a working standard of $1000 \mathrm{mg} \mathrm{L}^{-1} \mathrm{NO}_{3}{ }^{-}$.

Quality control was maintained with the use of certified samples that must fall within specified analytical windows $( \pm 15 \%)$ to meet USEPA requirements. Furthermore, all samples were analyzed in triplicate with a relative-percent-difference (RPD) of $<20 \%$ to qualify as acceptable under USEPA specifications [26]. Method detection limits were per USEPA conventions and are presented in Table 1.

Table 1. Method detection limits for target pollutants (metals and $\mathrm{NO}_{3}{ }^{-}$).

\begin{tabular}{cc}
\hline Constituents & Detection Limit \\
\hline $\mathrm{As}, \mathrm{Pb}$ & $0.025 \mathrm{mg} \mathrm{kg}^{-1}$ \\
$\mathrm{Ba}, \mathrm{Cd}, \mathrm{Co}, \mathrm{Cr}, \mathrm{Cu}, \mathrm{Mo}, \mathrm{Ni}, \mathrm{V}$ & $0.005 \mathrm{mg} \mathrm{kg}^{-1}$ \\
$\mathrm{Al}, \mathrm{Zn}$ & $0.1 \mathrm{mg} \mathrm{kg}^{-1}$ \\
$\mathrm{NO}_{3}{ }^{-}$as N & $0.1 \mathrm{mg} \mathrm{kg}^{-1}$ \\
\hline
\end{tabular}




\subsection{Statistical Analysis}

To identify associations among metals in lichen biomass and their possible sources, principal component analysis (PCA) and discriminant function analysis (DFA) were performed using multivariate analyses. Use of PCA to recognize associations among pollutants is a powerful tool when looking for similarities and patterns in groups of pollutants (e.g., metals, $\mathrm{NO}_{3}{ }^{-}-\mathrm{N}$ ). Discriminant function analysis is used to predict categorical dependent variable by one binary independent predictor variables across groups of pollutants. Minitab 17 statistical package software (Minitab, Inc., State

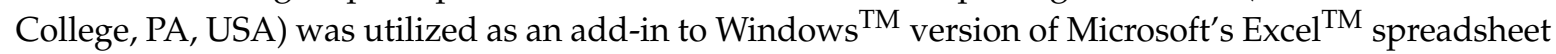
program with PCA and DFA performed using Varimax Normalized rotation.

In this study we aimed to identify air pollution within the Las Vegas Valley by using characteristic metal emission ratios [28]. To assess if air pollutants from the valley were detected at sampling sites, two sets of ratio analyses were undertaken: The first metal ratios were used to explore overall metal deposition between background and in valley samples. Such ratios from background locations were used to assess anthropogenic enhancement of metals and $\mathrm{NO}_{3}{ }^{-}$within the dataset (66 samples with six background samples; $n=72$ ). Ratio data were placed in geospatial context (N, E, S, W sectors) so that data could be interpreted according to locality. Other authors have reported that ratios greater than 1.0 are anthropogenically enhanced whereas values less than 1.0 are background in nature $[15,28]$. Therefore, for this study, ratios greater than 1 will be considered anthropogenically enhanced, those less than 1.0 will be considered background (non-enhanced). Ratio data was sample value compared with background average values.

The second metal ratios $(\mathrm{Cu} / \mathrm{Pb}, \mathrm{Co} / \mathrm{Cu}, \mathrm{Cr} / \mathrm{Pb}, \mathrm{Cd} / \mathrm{Pb}$ and $\mathrm{Cd} / \mathrm{Cu})$ utilized are known to be related to vehicle emissions $[15,28]$. Specific metal ratios related to vehicle emissions were performed because of their documented relationship between vehicle exhaust and air borne pollutants [28]. Data were used to quantify location ratios with in-valley emissions and windborne sources to recognize patterns within the valley and compare ground concentrations to windrose orientation. Finally, data were placed into a predictable (95\% probability) model of $\pm 1 \sigma$ of mean background values with data falling within this model considered to be anthropogenically enhanced across study sectors. Ratio data was sample value compared with background average values.

\section{Results}

\subsection{Background Locations}

Table 2 shows background concentrations for target analytes detected in lichen samples. This data provides a base for natural levels of target analytes in lichen biomass in areas not heavily impacted by anthropogenic activities. Samples used to measure background were collected from two (2) areas located 5 and $30 \mathrm{~km}$ from the Las Vegas Valley. These areas represent non-enhancement from the population centers (Figure 1). Data shows metals and $\mathrm{NO}_{3}{ }^{-}$background to be low when compared with other studies [29]. Sorted from highest to lowest mean values with $\mathrm{Al}>\mathrm{Zn}>\mathrm{Ba}>\mathrm{Pb}>\mathrm{Cu}>\mathrm{V}>$ $\mathrm{Cr}>\mathrm{Ni}>\mathrm{Co}>\mathrm{Cd}>\mathrm{As}>\mathrm{Mo}$ with $\mathrm{NO}_{3}{ }^{-}$averaging at $\sim 42 \mathrm{mg} \mathrm{kg}^{-1}$ (Table 2); levels considered low in lichen biomass according to Hauck et al. [29]. Arsenic, $\mathrm{Cd}$ and Mo were $<0.5 \mathrm{mg} \mathrm{kg}^{-1}$ while Ba was 77, Cr 6.2, Co 4.1, Ni 5.2, Pb 52, V 8.9, and Zn $96 \mathrm{mg} \mathrm{kg}^{-1}$, respectively. These levels are considered normal when compared to what Hauck et al. [29] reported in a similar study. 
Table 2. Summary statistics for metal and $\mathrm{NO}_{3}{ }^{-}$concentrations from 6 background collection sites.

\begin{tabular}{cccccccccccccc}
\hline $\begin{array}{c}\text { Sector } \\
\text { Location }\end{array}$ & Al & As & $\mathbf{B a}$ & $\mathbf{C d}$ & $\mathbf{C r}$ & $\mathbf{C o}$ & $\mathbf{C u}$ & $\mathbf{M o}$ & $\mathbf{N i}$ & $\mathbf{P b}$ & $\mathbf{V}$ & $\mathbf{Z n}$ & $\mathbf{N O}_{3}-$ \\
\hline \multirow{2}{*}{ SV1 } & 9802 & 0.18 & 104 & 0.9 & 8.7 & 5.1 & 15.8 & 0.02 & 8.4 & 77 & 14.2 & 176 & 20 \\
& $(1.5)$ & $(1.1)$ & $(1.3)$ & $(1.9)$ & $(1.4)$ & $(1.3)$ & $(1.7)$ & $(0.4)$ & $(1.6)$ & $(1.5)$ & $(1.6)$ & $(1.8)$ & $(0.48)$ \\
\hline \multirow{2}{*}{ SV2 } & 6330 & 0.25 & 87 & 0.4 & 5.8 & 3.5 & 9.6 & 0.06 & 5.5 & 41 & 6.5 & 72 & 36 \\
& $(1.0)$ & $(1.5)$ & $(1.1)$ & $(0.8)$ & $(0.9)$ & $(0.9)$ & $(1.0)$ & $(1.2)$ & $(1.1)$ & $(0.8)$ & $(0.7)$ & $(0.7)$ & $(0.9)$ \\
\hline \multirow{2}{*}{ SV3 } & 5811 & 0.1 & 78 & 0.8 & 5.8 & 3.6 & 11.6 & 0.04 & 5.6 & 49 & 7.9 & 100 & 29 \\
& $(0.9)$ & $(0.1)$ & $(1.0)$ & $(1.7)$ & $(0.9)$ & $(0.9)$ & $(1.2)$ & $(0.8)$ & $(1.1)$ & $(0.9)$ & $(0.9)$ & $(1.0)$ & $(0.7)$ \\
\hline \multirow{2}{*}{ SV4 } & 5268 & 0.18 & 61 & 0.1 & 5.2 & 3.1 & 9.7 & 0.03 & 5.0 & 65 & 6.5 & 114 & 32 \\
& $(0.8)$ & $(1.1)$ & $(0.8)$ & $(0.2)$ & $(0.8)$ & $(0.8)$ & $(1.0)$ & $(0.6)$ & $(1.0)$ & $(1.2)$ & $(0.7)$ & $(1.2)$ & $(0.8)$ \\
\hline \multirow{2}{*}{25} & 6218 & 0.17 & 72 & 0.5 & 6.8 & 5.4 & 5.4 & 0.04 & 3.94 & 59 & 10.0 & 74 & 66 \\
& $(1.0)$ & $(1.0)$ & $(0.9)$ & $(1.0)$ & $(1.1)$ & $(1.3)$ & $(0.6)$ & $(0.8)$ & $(0.8)$ & $(1.1)$ & $(1.1)$ & $(0.8)$ & $(1.6)$ \\
\hline \multirow{2}{*}{26} & 4676 & 0.16 & 61 & 0.3 & 5.0 & 3.7 & 5.2 & 0.08 & 2.72 & 24 & 8.3 & 41 & 69 \\
& $(0.7)$ & $(0.9)$ & $(0.8)$ & $(0.6)$ & $(0.8)$ & $(0.9)$ & $(0.5)$ & $(1.6)$ & $(0.5)$ & $(0.5)$ & $(0.9)$ & $(0.4)$ & $(1.6)$ \\
\hline Mean & 6351 & 0.17 & 77.27 & 0.48 & 6.23 & 4.07 & 9.53 & 0.05 & 5.20 & 52.34 & 8.90 & 96.20 & 41.95 \\
STDEV & 1800 & 0.05 & 17 & 0.32 & 1.37 & 0.94 & 4.00 & 0.02 & 1.91 & 19 & 2.90 & 47 & 20 \\
Max & 9802 & 0 & 104 & 1 & 9 & 5 & 16 & 0 & 8 & 77 & 14 & 176 & 69 \\
Min & 4676 & 0 & 61 & 0 & 5 & 3 & 5 & 0 & 3 & 24 & 6 & 41 & 20 \\
\hline
\end{tabular}

Note: Values reported in $\mathrm{mg} \mathrm{kg}^{-1}$ in lichen; STDEV: standard deviation. $\pm 1 \sigma$ of background mean; ( ) are ratios with average background value; SV: Sandy Valley.

\subsection{Las Vegas Valley Locations}

The number of analytical samples collected for background $(n=6)$ is not sufficient to have a final conclusion for lichen outside the entire valley however, some observations can be drawn for instance, a comparison between the results obtained for samples collected from background sites were lower than the majority of samples collected from within the valley. Results indicate that samples from the valley are a good baseline of pollutants in lichens. Furthermore, lichen collected within the valley contained higher concentrations of target pollutants suggesting that the accumulation of pollution is likely anthropogenic in nature (e.g., industries, vehicular traffic).

Trace elements ( $\mathrm{Al}, \mathrm{As}, \mathrm{Ba}, \mathrm{Cd}, \mathrm{Cr}, \mathrm{Co}, \mathrm{Cu}, \mathrm{Mo}, \mathrm{Ni}, \mathrm{Pb}, \mathrm{V}$ and $\mathrm{Zn}$ ) and $\mathrm{NO}_{3}{ }^{-}$data reveal two distinct areas of enrichment; East and South sectors (Tables 3-6, Figure 2). When compared with background values, data show that the East (E) and South (S) sectors were greater than $1 \sigma$ of background whereas the North $(\mathrm{N})$ and West $(\mathrm{W})$ sectors were greater than or equal to background values. Within the $\mathrm{S}$ sector several locations show $\mathrm{As}, \mathrm{Cr}, \mathrm{Cu}$ and $\mathrm{Pb}$ greater than background mean, with some locations greater than 1.5 times background mean (i.e., highest values for As at 2.2, 9.0 Cr, $16 \mathrm{Cu}, 73 \mathrm{~Pb} \mathrm{mg} \mathrm{kg}-1$, at $12.9,1.44,1.7$ and $1.4 \times$ background, respectively). Results from the E sector contained higher values than detected in the $\mathrm{S}$ sector with As measuring at 11.2, $\mathrm{Cd}$ 3.6, $\mathrm{Cr}$ 39, $\mathrm{Cu}$ 89, $\mathrm{Co} \mathrm{16,} \mathrm{Mo} \mathrm{1.02,} \mathrm{Pb} \mathrm{81,} \mathrm{V} \mathrm{48,} \mathrm{and} \mathrm{Zn}$ at $681 \mathrm{mg} \mathrm{kg}^{-1}$, respectively. A few locations in the $\mathrm{S}$ sector measured $9 \times$ greater than background for $\mathrm{Cu}, 6 \times \mathrm{Cr}, 7.5 \times \mathrm{Cd}$ and $65 \times$ greater than background however, only a few locations contained detectable $\mathrm{As}$ (3) and $\mathrm{Cd}$ (5). A few localities also contained significant levels of $\mathrm{Co}, \mathrm{Mo}, \mathrm{Ni}, \mathrm{Pb}, \mathrm{V}$ and $\mathrm{Zn}(5.3 \mathrm{Co}, 0.36 \mathrm{Mo}, 5.5 \mathrm{Ni}, 65 \mathrm{~Pb}$, $13.2 \mathrm{~V}$ and $117 \mathrm{Zn} \mathrm{mg} \mathrm{kg}^{-1}$ ), whereas the remainder of locations measured greater than, or equal to, background levels. 
Table 3. Data for metals and $\mathrm{NO}_{3}{ }^{-}$concentrations in E sector.

\begin{tabular}{|c|c|c|c|c|c|c|c|c|c|c|c|c|c|}
\hline $\begin{array}{l}\text { Sector } \\
\text { Location }\end{array}$ & Al & As & Ba & $\mathrm{Cd}$ & $\mathrm{Cr}$ & Co & $\mathrm{Cu}$ & Mo & $\mathbf{N i}$ & $\mathrm{Pb}$ & $\mathbf{V}$ & $\mathrm{Zn}$ & $\mathrm{NO}_{3}{ }^{-}$ \\
\hline 56 & $\begin{array}{l}15,255 \\
(2.4)\end{array}$ & $\begin{array}{l}- \\
(-)\end{array}$ & $\begin{array}{l}153 \\
(2.0)\end{array}$ & $\begin{array}{l}8.00 \\
(16)\end{array}$ & $\begin{array}{c}16 \\
(2.6)\end{array}$ & $\begin{array}{c}59 \\
(14.5)\end{array}$ & $\begin{array}{c}25 \\
(2.6)\end{array}$ & (-) & $\begin{array}{c}123 \\
(23.7)\end{array}$ & $\begin{array}{c}268 \\
(5.1)\end{array}$ & $\begin{array}{l}161 \\
(18)\end{array}$ & $\begin{array}{c}681 \\
(7.1)\end{array}$ & $\begin{array}{c}18 \\
(0.4)\end{array}$ \\
\hline 57 & $\begin{array}{l}5671 \\
(0.9)\end{array}$ & $\begin{array}{l}- \\
(-)\end{array}$ & $\begin{array}{c}81 \\
(1.0)\end{array}$ & $\begin{array}{l}0.34 \\
(0.7)\end{array}$ & $\begin{array}{l}5.7 \\
(0.9)\end{array}$ & $\begin{array}{c}2.6 \\
(0.6)\end{array}$ & $\begin{array}{l}11.0 \\
(1.2)\end{array}$ & $\begin{array}{l}- \\
(-)\end{array}$ & $\begin{array}{c}5.3 \\
(1.0)\end{array}$ & $\begin{array}{c}21 \\
(0.1)\end{array}$ & $\begin{array}{c}6.0 \\
(0.7)\end{array}$ & $\begin{array}{c}35 \\
(0.4)\end{array}$ & $\begin{array}{c}53 \\
(1.3)\end{array}$ \\
\hline 58 & $\begin{array}{l}7028 \\
(1.1)\end{array}$ & $\begin{array}{c}1.6 \\
(9.2)\end{array}$ & $\begin{array}{c}256 \\
(3.3) \\
\end{array}$ & $\begin{array}{l}0.34 \\
(0.7) \\
\end{array}$ & $\begin{array}{c}6.4 \\
(1.0) \\
\end{array}$ & $\begin{array}{c}3.0 \\
(0.7) \\
\end{array}$ & $\begin{array}{l}15.3 \\
(1.6)\end{array}$ & $\begin{array}{l}- \\
(-)\end{array}$ & $\begin{array}{c}5.5 \\
(1.1) \\
\end{array}$ & $\begin{array}{c}27 \\
(0.5)\end{array}$ & $\begin{array}{c}5.2 \\
(0.6)\end{array}$ & $\begin{array}{c}40 \\
(0.4) \\
\end{array}$ & $\begin{array}{c}9.7 \\
(0.2)\end{array}$ \\
\hline 59 & $\begin{array}{l}71,180 \\
(11.2)\end{array}$ & $\begin{array}{c}- \\
(-)\end{array}$ & $\begin{array}{c}3.6 \\
(0.0)\end{array}$ & $\begin{array}{l}3.60 \\
(7.4)\end{array}$ & $\begin{array}{c}71 \\
(11.4)\end{array}$ & $\begin{array}{c}31 \\
(7.6)\end{array}$ & $\begin{array}{c}14 \\
(1.5)\end{array}$ & $(-)$ & $\begin{array}{c}57 \\
(11.0)\end{array}$ & $\begin{array}{c}78 \\
(1.5)\end{array}$ & $\begin{array}{c}87 \\
(9.8)\end{array}$ & $\begin{array}{c}412 \\
(4.3)\end{array}$ & $\begin{array}{l}14.9 \\
(0.4)\end{array}$ \\
\hline 60 & $\begin{array}{l}11,112 \\
(1.7)\end{array}$ & $\begin{array}{c}- \\
(-)\end{array}$ & $\begin{array}{c}31 \\
(0.4)\end{array}$ & $\begin{array}{l}0.30 \\
(0.6)\end{array}$ & $\begin{array}{l}16.3 \\
(2.6)\end{array}$ & $\begin{array}{c}6.0 \\
(1.5)\end{array}$ & $\begin{array}{c}30 \\
(3.1)\end{array}$ & $\begin{array}{c}- \\
(-)\end{array}$ & $\begin{array}{l}11.3 \\
(2.2)\end{array}$ & $\begin{array}{c}41 \\
(0.8)\end{array}$ & $\begin{array}{l}19.6 \\
(2.2)\end{array}$ & $\begin{array}{c}59 \\
(0.6)\end{array}$ & $\begin{array}{c}- \\
(-)\end{array}$ \\
\hline 61 & $\begin{array}{l}42,714 \\
(6.7)\end{array}$ & $(-)$ & $\begin{array}{l}559 \\
(7.2)\end{array}$ & $\begin{array}{l}1.30 \\
(2.7)\end{array}$ & $\begin{array}{c}39 \\
(6.2) \\
\end{array}$ & $\begin{array}{l}16.0 \\
(3.9)\end{array}$ & $\begin{array}{c}89 \\
(9.3)\end{array}$ & $\begin{array}{l}- \\
(-)\end{array}$ & $\begin{array}{c}31 \\
(6.0)\end{array}$ & $\begin{array}{c}81 \\
(1.5)\end{array}$ & $\begin{array}{c}48 \\
(5.4)\end{array}$ & $\begin{array}{l}196 \\
(2.0)\end{array}$ & $\begin{array}{c}50 \\
(1.2) \\
\end{array}$ \\
\hline 62 & $\begin{array}{l}3980 \\
(0.6)\end{array}$ & $\begin{array}{c}- \\
(-)\end{array}$ & $\begin{array}{c}67 \\
(0.9)\end{array}$ & $\begin{array}{l}0.18 \\
(0.4)\end{array}$ & $\begin{array}{c}4.2 \\
(0.7)\end{array}$ & $\begin{array}{c}1.9 \\
(0.5)\end{array}$ & $\begin{array}{c}7.1 \\
(0.7)\end{array}$ & $\begin{array}{l}- \\
(-)\end{array}$ & $\begin{array}{c}3.7 \\
(0.7)\end{array}$ & $\begin{array}{l}19.7 \\
(0.4)\end{array}$ & $\begin{array}{c}4.5 \\
(0.5)\end{array}$ & $\begin{array}{c}23 \\
(0.2)\end{array}$ & $\begin{array}{c}2 \\
(0.0)\end{array}$ \\
\hline 63 & $\begin{array}{l}3772 \\
(0.6)\end{array}$ & $\begin{array}{c}5.4 \\
(31.2)\end{array}$ & $\begin{array}{l}276 \\
(3.6)\end{array}$ & $\begin{array}{l}0.15 \\
(0.3)\end{array}$ & $\begin{array}{c}4.1 \\
(0.7)\end{array}$ & $\begin{array}{c}3.8 \\
(0.9)\end{array}$ & $\begin{array}{c}5.7 \\
(0.6)\end{array}$ & $\begin{array}{l}0.40 \\
(8.9)\end{array}$ & $\begin{array}{c}3.8 \\
(0.7)\end{array}$ & $\begin{array}{c}29 \\
(0.6)\end{array}$ & $\begin{array}{c}7.0 \\
(0.8)\end{array}$ & $\begin{array}{c}28 \\
(0.3)\end{array}$ & $\begin{array}{c}1.2 \\
(0.0)\end{array}$ \\
\hline 64 & $\begin{array}{l}3162 \\
(0.6) \\
\end{array}$ & $\begin{array}{c}2.07 \\
(11.9) \\
\end{array}$ & $\begin{array}{c}292 \\
(3.8) \\
\end{array}$ & $\begin{array}{c}- \\
(-) \\
\end{array}$ & $\begin{array}{c}4.4 \\
(0.7) \\
\end{array}$ & $\begin{array}{c}3.6 \\
(0.9) \\
\end{array}$ & $\begin{array}{l}12.0 \\
(1.3) \\
\end{array}$ & $\begin{array}{l}0.41 \\
(9.1) \\
\end{array}$ & $\begin{array}{c}4.3 \\
(0.8) \\
\end{array}$ & $\begin{array}{c}30 \\
(0.6) \\
\end{array}$ & $\begin{array}{c}7.2 \\
(0.8) \\
\end{array}$ & $\begin{array}{c}38 \\
(0.4) \\
\end{array}$ & $\begin{array}{l}0.45 \\
(0.0) \\
\end{array}$ \\
\hline 65 & $\begin{array}{l}4422 \\
(0.7)\end{array}$ & $(-)$ & $\begin{array}{c}236 \\
(3.1)\end{array}$ & $(-)$ & $\begin{array}{c}6.0 \\
(1.0)\end{array}$ & $\begin{array}{c}4.6 \\
(1.1)\end{array}$ & $\begin{array}{c}3.6 \\
(0.4) \\
\end{array}$ & $\begin{array}{l}0.52 \\
(11)\end{array}$ & $\begin{array}{c}4.8 \\
(0.9)\end{array}$ & $\begin{array}{c}42 \\
(0.8)\end{array}$ & $\begin{array}{c}9.8 \\
(1.1)\end{array}$ & $\begin{array}{c}32 \\
(0.3)\end{array}$ & $\begin{array}{l}0.78 \\
(0.0)\end{array}$ \\
\hline 66 & $\begin{array}{l}4492 \\
(0.7)\end{array}$ & $\begin{array}{c}6.2 \\
(35.8) \\
\end{array}$ & $\begin{array}{l}183 \\
(2.4)\end{array}$ & $\begin{array}{c}- \\
(-)\end{array}$ & $\begin{array}{c}5.8 \\
(0.9)\end{array}$ & $\begin{array}{c}4.4 \\
(1.1)\end{array}$ & $\begin{array}{c}4.1 \\
(0.4)\end{array}$ & $\begin{array}{l}0.53 \\
(11)\end{array}$ & $\begin{array}{c}4.4 \\
(0.9)\end{array}$ & $\begin{array}{c}88 \\
(1.7)\end{array}$ & $\begin{array}{c}9.7 \\
(1.1)\end{array}$ & $\begin{array}{c}36 \\
(0.4)\end{array}$ & $\begin{array}{l}1.35 \\
(0.0)\end{array}$ \\
\hline 67 & $\begin{array}{l}4076 \\
(0.6)\end{array}$ & $\begin{array}{c}- \\
(-)\end{array}$ & $\begin{array}{c}212 \\
(2.7)\end{array}$ & $\begin{array}{c}- \\
(-)\end{array}$ & $\begin{array}{c}4.3 \\
(0.7)\end{array}$ & $\begin{array}{c}4.3 \\
(1.0)\end{array}$ & $\begin{array}{c}- \\
(-)\end{array}$ & $\begin{array}{c}- \\
(-)\end{array}$ & $\begin{array}{c}5.0 \\
(1.0)\end{array}$ & $\begin{array}{c}56 \\
(1.1)\end{array}$ & $\begin{array}{c}8.9 \\
(1.0)\end{array}$ & $\begin{array}{c}31 \\
(0.3)\end{array}$ & $\begin{array}{l}0.57 \\
(0.0)\end{array}$ \\
\hline 68 & $\begin{array}{l}4061 \\
(0.6)\end{array}$ & $\begin{array}{c}11.2 \\
(64.5)\end{array}$ & $\begin{array}{c}151 \\
(2.0)\end{array}$ & $\begin{array}{l}0.13 \\
(0.3)\end{array}$ & $\begin{array}{l}18.9 \\
(3.0)\end{array}$ & $\begin{array}{c}6.4 \\
(1.6)\end{array}$ & $\begin{array}{l}11.9 \\
(1.3)\end{array}$ & $\begin{array}{l}1.02 \\
(22)\end{array}$ & $\begin{array}{c}8.0 \\
(1.5)\end{array}$ & $\begin{array}{c}33 \\
(0.6)\end{array}$ & $\begin{array}{l}14.2 \\
(1.6)\end{array}$ & $\begin{array}{c}51 \\
(0.5)\end{array}$ & $\begin{array}{l}0.54 \\
(0.0)\end{array}$ \\
\hline Mean & 13,917 & 2.0 & 192 & 1.1 & 16 & 11 & 18 & 0.22 & 21 & 63 & 30 & 128 & 12 \\
\hline STDEV & 20,256 & 3.5 & 145 & 2.3 & 19 & 16 & 23 & & 34 & 66 & 46 & 199 & 19 \\
\hline Max & 71,180 & 11.2 & 559 & 8 & 71 & 59 & 89 & 1.02 & 123 & 268 & 161 & 681 & 53 \\
\hline Min & 3162 & - & 3.6 & - & 4.1 & 1.9 & - & 0.00 & 3.7 & 19.7 & 6.0 & 28 & - \\
\hline
\end{tabular}

Note: Values reported in $\mathrm{mg} \mathrm{kg}^{-1}$ in lichen; STDEV: standard deviation. $\pm 1 \sigma$ of background mean; - Nil; ( ) are pollutant ratios with ambient background averages.

Table 4. Data for metals and $\mathrm{NO}_{3}{ }^{-}$concentrations in $\mathrm{S}$ sector.

\begin{tabular}{cccccccccccccc}
\hline $\begin{array}{c}\text { Sector } \\
\text { Location }\end{array}$ & $\mathbf{A l}$ & $\mathbf{A s}$ & $\mathbf{B a}$ & $\mathbf{C d}$ & $\mathbf{C r}$ & $\mathbf{C o}$ & $\mathbf{C u}$ & $\mathbf{M o}$ & $\mathbf{N i}$ & $\mathbf{P b}$ & $\mathbf{V}$ & $\mathbf{Z n}$ & $\mathbf{N O}_{3}^{-}$ \\
\hline \multirow{2}{*}{1} & 5542 & 0.19 & 103 & 0.38 & 7.7 & 3.9 & 11.2 & 0.26 & 7.7 & 63 & 6.7 & 42 & 42 \\
& $(0.9)$ & $(1.1)$ & $(1.3)$ & $(0.8)$ & $(1.2)$ & $(1.9)$ & $(1.2)$ & $(5.7)$ & $(1.5)$ & $(1.2)$ & $(0.7)$ & $(0.4)$ & $(1.0)$ \\
\hline \multirow{2}{*}{2} & 5205 & 1.4 & 137 & 0.25 & 7.7 & 3.5 & 12.5 & 0.35 & 6.5 & 46 & 11.3 & 51 & 33 \\
& $(0.8)$ & $(7.8)$ & $(1.8)$ & $(0.5)$ & $(1.2)$ & $(0.8)$ & $(1.3)$ & $(7.8)$ & $(1.3)$ & $(0.9)$ & $(1.3)$ & $(0.5)$ & $(0.8)$ \\
\hline \multirow{2}{*}{3} & 6430 & - & 142 & 0.40 & 6.6 & 4.3 & 14.1 & - & 7.2 & 50 & 10.6 & 46 & 38 \\
& $(1.0)$ & $(-)$ & $(1.8)$ & $(0.8)$ & $(1.1)$ & $(1.0)$ & $(1.5)$ & $(-)$ & $(1.4)$ & $(0.9)$ & $(1.2)$ & $(0.5)$ & $(0.9)$ \\
\hline \multirow{2}{*}{4} & 6898 & - & 174 & 0.22 & 8.2 & 5.2 & 8.5 & - & 6.4 & 54 & 9.9 & 55 & 28 \\
& $(1.1)$ & $(-)$ & $(2.3)$ & $(0.5)$ & $(1.3)$ & $(1.3)$ & $(0.9)$ & $(-)$ & $(1.2)$ & $(1.0)$ & $(1.1)$ & $(0.6)$ & $(0.7)$ \\
\hline \multirow{2}{*}{5} & 5132 & 2.2 & 143 & 0.36 & 4.7 & 3.9 & 16.6 & - & 5.4 & 37 & 7.4 & 44 & 30 \\
& $(0.8)$ & $(13)$ & $(1.8)$ & $(0.8)$ & $(0.8)$ & $(1.0)$ & $(1.7)$ & $(-)$ & $(1.0)$ & $(0.7)$ & $(0.8)$ & $(0.5)$ & $(0.7)$ \\
\hline \multirow{2}{*}{6} & 4791 & - & 126 & 0.09 & 5.8 & 3.7 & 10.1 & - & 4.6 & 42 & 0.3 & 39 & 53 \\
& $(0.8)$ & $(-)$ & $(1.6)$ & $(0.2)$ & $(0.9)$ & $(0.9)$ & $(1.1)$ & $(-)$ & $(0.9)$ & $(0.8)$ & $(1.0)$ & $(0.4)$ & $(1.3)$ \\
\hline \multirow{2}{*}{7} & 7300 & - & 144 & - & 8.6 & 5.4 & 10.7 & - & 7.2 & 51 & 11.6 & 40 & 63 \\
& $(1.1)$ & $(-)$ & $(1.9)$ & $(-)$ & $(1.4)$ & $(1.3)$ & $(1.1)$ & $(-)$ & $(1.4)$ & $(1.0)$ & $(1.3)$ & $(0.4)$ & $(1.5)$ \\
\hline
\end{tabular}


Table 4. Cont.

\begin{tabular}{|c|c|c|c|c|c|c|c|c|c|c|c|c|c|}
\hline $\begin{array}{l}\text { Sector } \\
\text { Location }\end{array}$ & Al & As & Ва & $\mathrm{Cd}$ & $\mathrm{Cr}$ & Co & $\mathrm{Cu}$ & Mo & $\mathbf{N i}$ & $\mathrm{Pb}$ & $\mathbf{V}$ & $\mathrm{Zn}$ & $\mathrm{NO}_{3}{ }^{-}$ \\
\hline 8 & $\begin{array}{l}4567 \\
(0.7)\end{array}$ & $\begin{array}{c}- \\
(-)\end{array}$ & $\begin{array}{c}120 \\
(1.5)\end{array}$ & $\begin{array}{l}0.28 \\
(0.6)\end{array}$ & $\begin{array}{c}5.3 \\
(0.8)\end{array}$ & $\begin{array}{c}3.8 \\
(0.9)\end{array}$ & $\begin{array}{c}9.7 \\
(1.0)\end{array}$ & $\begin{array}{l}- \\
(-)\end{array}$ & $\begin{array}{c}5.0 \\
(1.0)\end{array}$ & $\begin{array}{c}49 \\
(0.9)\end{array}$ & $\begin{array}{c}7.5 \\
(0.8)\end{array}$ & $\begin{array}{c}39 \\
(0.4)\end{array}$ & $\begin{array}{c}- \\
(-)\end{array}$ \\
\hline 9 & $\begin{array}{l}7390 \\
(1.2)\end{array}$ & $(-$ & $\begin{array}{l}164 \\
(2.1)\end{array}$ & $\begin{array}{l}0.11 \\
(0.2)\end{array}$ & $\begin{array}{c}9.0 \\
(1.5)\end{array}$ & $\begin{array}{c}6.2 \\
(1.5)\end{array}$ & $\begin{array}{l}11.3 \\
(1.2)\end{array}$ & $\begin{array}{l}- \\
(-)\end{array}$ & $\begin{array}{c}8.5 \\
(1.6)\end{array}$ & $\begin{array}{c}52 \\
(1.0)\end{array}$ & $\begin{array}{l}13.2 \\
(1.5)\end{array}$ & $\begin{array}{c}55 \\
(0.6)\end{array}$ & $\begin{array}{c}74 \\
(1.8)\end{array}$ \\
\hline 10 & $\begin{array}{l}1684 \\
(0.3)\end{array}$ & $\begin{array}{l}- \\
(-)\end{array}$ & $\begin{array}{l}106 \\
(1.4)\end{array}$ & $\begin{array}{l}- \\
(-)\end{array}$ & $\begin{array}{c}3.8 \\
(0.6)\end{array}$ & $\begin{array}{l}1.8 \\
(0.4)\end{array}$ & $\begin{array}{c}5.7 \\
(0.6)\end{array}$ & $\begin{array}{c}0.46 \\
(10.1)\end{array}$ & $\begin{array}{c}0.6 \\
(0.1)\end{array}$ & $\begin{array}{c}36 \\
(0.7)\end{array}$ & $\begin{array}{c}5.9 \\
(0.7)\end{array}$ & $\begin{array}{c}65 \\
(0.7)\end{array}$ & $\begin{array}{c}14 \\
(0.3)\end{array}$ \\
\hline 11 & $\begin{array}{l}2079 \\
(0.3)\end{array}$ & $\begin{array}{c}1.1 \\
(6.1)\end{array}$ & $\begin{array}{c}86 \\
(1.1)\end{array}$ & $\begin{array}{l}0.20 \\
(0.4)\end{array}$ & $\begin{array}{c}3.8 \\
(0.6)\end{array}$ & $\begin{array}{c}2.4 \\
(0.6)\end{array}$ & $\begin{array}{l}10.4 \\
(1.1)\end{array}$ & $\begin{array}{c}0.66 \\
(14.7)\end{array}$ & $\begin{array}{c}1.9 \\
(0.4)\end{array}$ & $\begin{array}{c}73 \\
(1.4)\end{array}$ & $\begin{array}{c}6.5 \\
(0.7) \\
\end{array}$ & $\begin{array}{c}60 \\
(0.6)\end{array}$ & $\begin{array}{c}75 \\
(1.8)\end{array}$ \\
\hline 12 & $\begin{array}{l}3770 \\
(0.6)\end{array}$ & $\begin{array}{c}0.9 \\
(5.1)\end{array}$ & $\begin{array}{c}291 \\
(3.8)\end{array}$ & $(-)$ & $\begin{array}{c}6.5 \\
(1.1)\end{array}$ & $\begin{array}{c}4.4 \\
(1.1)\end{array}$ & $\begin{array}{l}15.2 \\
(1.6)\end{array}$ & $\begin{array}{c}- \\
(-)\end{array}$ & $\begin{array}{c}2.5 \\
(0.5)\end{array}$ & $\begin{array}{c}63 \\
(1.2)\end{array}$ & $\begin{array}{l}12.6 \\
(1.4)\end{array}$ & $\begin{array}{c}85 \\
(0.9)\end{array}$ & $\begin{array}{c}39 \\
(0.9)\end{array}$ \\
\hline 13 & $\begin{array}{l}2048 \\
(0.3)\end{array}$ & $\begin{array}{c}2.1 \\
(12)\end{array}$ & $\begin{array}{c}80 \\
(1.0)\end{array}$ & $\begin{array}{l}0.10 \\
(0.2)\end{array}$ & $\begin{array}{c}3.6 \\
(0.6)\end{array}$ & $\begin{array}{c}3.1 \\
(0.8)\end{array}$ & $\begin{array}{c}5.6 \\
(0.6)\end{array}$ & $(-$ & $\begin{array}{l}1.8 \\
(0.3)\end{array}$ & $\begin{array}{c}43 \\
(0.8)\end{array}$ & $\begin{array}{c}7.6 \\
(0.9) \\
\end{array}$ & $\begin{array}{c}51 \\
(0.5)\end{array}$ & $\begin{array}{l}102 \\
(2.4)\end{array}$ \\
\hline 14 & 52180.8 & $\begin{array}{c}- \\
(-)\end{array}$ & $\begin{array}{l}130 \\
(1.7)\end{array}$ & $\begin{array}{l}- \\
(-)\end{array}$ & $\begin{array}{c}6.9 \\
(1.1)\end{array}$ & $\begin{array}{l}10.6 \\
(2.6)\end{array}$ & $\begin{array}{c}5.5 \\
(0.6)\end{array}$ & $\begin{array}{l}- \\
(-)\end{array}$ & $\begin{array}{l}15.8 \\
(3.0)\end{array}$ & $\begin{array}{c}35 \\
(0.7)\end{array}$ & $\begin{array}{l}12.3 \\
(1.4)\end{array}$ & $\begin{array}{c}53 \\
(0.6)\end{array}$ & $\begin{array}{c}26 \\
(0.6)\end{array}$ \\
\hline 15 & $\begin{array}{l}2394 \\
(0.4)\end{array}$ & $\begin{array}{c}0.7 \\
(3.8)\end{array}$ & $\begin{array}{c}50 \\
(0.6)\end{array}$ & $\begin{array}{l}0.06 \\
(0.1)\end{array}$ & $\begin{array}{c}1.8 \\
(0.3)\end{array}$ & $\begin{array}{c}2.4 \\
(0.6)\end{array}$ & $\begin{array}{c}8.8 \\
(0.9)\end{array}$ & $\begin{array}{l}0.06 \\
(1.3)\end{array}$ & $\begin{array}{c}2.9 \\
(0.6)\end{array}$ & $\begin{array}{c}11 \\
(0.2)\end{array}$ & $\begin{array}{c}4.1 \\
(0.5)\end{array}$ & $\begin{array}{c}15 \\
(0.2)\end{array}$ & $\begin{array}{l}129 \\
(3.1)\end{array}$ \\
\hline 16 & $\begin{array}{l}5188 \\
(0.8)\end{array}$ & $\begin{array}{l}- \\
(-)\end{array}$ & $\begin{array}{l}354 \\
(4.6)\end{array}$ & $\begin{array}{l}- \\
(-)\end{array}$ & $\begin{array}{c}7.0 \\
(1.1)\end{array}$ & $\begin{array}{c}8.1 \\
(2.0)\end{array}$ & $\begin{array}{c}6.8 \\
(0.7)\end{array}$ & $\begin{array}{l}0.19 \\
(4.2)\end{array}$ & $\begin{array}{c}3.8 \\
(0.7)\end{array}$ & $\begin{array}{c}10 \\
(0.2)\end{array}$ & $\begin{array}{l}15.9 \\
(1.8)\end{array}$ & $\begin{array}{c}69 \\
(0.7)\end{array}$ & $\begin{array}{c}60 \\
(1.4)\end{array}$ \\
\hline 17 & $\begin{array}{l}4360 \\
(0.7)\end{array}$ & $\begin{array}{c}- \\
(-)\end{array}$ & $\begin{array}{c}93 \\
(1.2)\end{array}$ & $\begin{array}{l}0.14 \\
(0.3)\end{array}$ & $\begin{array}{c}5.7 \\
(0.9)\end{array}$ & $\begin{array}{c}3.6 \\
(0.9)\end{array}$ & $\begin{array}{c}8.9 \\
(0.9)\end{array}$ & $\begin{array}{l}0.21 \\
(4.7)\end{array}$ & $\begin{array}{c}4.3 \\
(0.8)\end{array}$ & $\begin{array}{c}31 \\
(0.6)\end{array}$ & $\begin{array}{c}8.8 \\
(1.0) \\
\end{array}$ & $\begin{array}{c}42 \\
(0.4)\end{array}$ & $\begin{array}{c}51 \\
(1.2)\end{array}$ \\
\hline 18 & $\begin{array}{l}4694 \\
(0.7)\end{array}$ & $\overline{-}$ & $\begin{array}{c}97 \\
(1.3)\end{array}$ & $\begin{array}{c}- \\
(-)\end{array}$ & $\begin{array}{c}6.3 \\
(1.0)\end{array}$ & $\begin{array}{c}3.8 \\
(0.9)\end{array}$ & $\begin{array}{l}11.6 \\
(1.2)\end{array}$ & $\begin{array}{l}0.45 \\
(9.9)\end{array}$ & $\begin{array}{c}5.0 \\
(1.0)\end{array}$ & $\begin{array}{c}40 \\
(0.8)\end{array}$ & $\begin{array}{l}10.6 \\
(1.2)\end{array}$ & $\begin{array}{c}49 \\
(0.5)\end{array}$ & $\begin{array}{c}53 \\
(1.3)\end{array}$ \\
\hline 19 & $\begin{array}{l}6752 \\
(1.1)\end{array}$ & $\begin{array}{l}- \\
(-)\end{array}$ & $\begin{array}{c}113 \\
(1.5)\end{array}$ & $\begin{array}{l}0.26 \\
(0.5)\end{array}$ & $\begin{array}{c}8.8 \\
(1.4)\end{array}$ & $\begin{array}{c}5.1 \\
(1.3)\end{array}$ & $\begin{array}{l}17.3 \\
(1.8)\end{array}$ & $\begin{array}{c}- \\
(-)\end{array}$ & $\begin{array}{c}7.0 \\
(1.3)\end{array}$ & $\begin{array}{c}46 \\
(0.9)\end{array}$ & $\begin{array}{l}11.1 \\
(1.2)\end{array}$ & $\begin{array}{c}56 \\
(0.6)\end{array}$ & $\begin{array}{c}- \\
(-)\end{array}$ \\
\hline 20 & $\begin{array}{l}4054 \\
(0.6)\end{array}$ & $\begin{array}{l}- \\
(-)\end{array}$ & $\begin{array}{c}78 \\
(1.0)\end{array}$ & $\begin{array}{c}0.11 \\
(0.2)\end{array}$ & $\begin{array}{c}5.1 \\
(0.8)\end{array}$ & $\begin{array}{c}2.8 \\
(0.7)\end{array}$ & $\begin{array}{c}6.8 \\
(0.7)\end{array}$ & $\begin{array}{l}- \\
\end{array}$ & $\begin{array}{c}4.0 \\
(0.8)\end{array}$ & $\begin{array}{c}17 \\
(0.3)\end{array}$ & $\begin{array}{c}6.3 \\
(0.7)\end{array}$ & $\begin{array}{c}27 \\
(0.3)\end{array}$ & $\begin{array}{c}68 \\
(1.6)\end{array}$ \\
\hline 21 & $\begin{array}{l}3361 \\
(0.5)\end{array}$ & $\begin{array}{l}- \\
(-)\end{array}$ & $\begin{array}{c}71 \\
(0.9)\end{array}$ & $\begin{array}{l}- \\
(-)\end{array}$ & $\begin{array}{c}5.4 \\
(0.9)\end{array}$ & $\begin{array}{c}3.2 \\
(0.8)\end{array}$ & $\begin{array}{c}- \\
(-)\end{array}$ & $\begin{array}{l}0.32 \\
(7.1)\end{array}$ & $\begin{array}{c}1.6 \\
(0.3)\end{array}$ & $\begin{array}{c}33 \\
(0.6)\end{array}$ & $\begin{array}{c}7.6 \\
(0.9)\end{array}$ & $\begin{array}{c}42 \\
(0.4)\end{array}$ & $\begin{array}{c}79 \\
(1.9)\end{array}$ \\
\hline Mean & 4708 & 0.4 & 133 & 0.15 & 6.1 & 4.3 & 9.9 & 0.15 & 5.2 & 42 & 8.9 & 49 & 50 \\
\hline STDEV & 1721 & 0.7 & 71 & 0.14 & 1.9 & 2.0 & 4.1 & 0.20 & 3.3 & 16 & 3.5 & 15 & 32 \\
\hline Max & 7390 & 2.2 & 354 & 0.40 & 9.0 & 10.6 & 17.3 & 0.66 & 15.8 & 73 & 15.9 & 85 & 129 \\
\hline Min & 1684 & - & 50 & - & 1.8 & 1.8 & - & - & 0.6 & 10 & 0.3 & 15 & - \\
\hline
\end{tabular}

Note: Values reported in $\mathrm{mg} \mathrm{kg}^{-1}$ in lichen; STDEV: standard deviation. $\pm 1 \sigma$ of background mean; - Nil; ( ) are pollutant ratios with ambient background averages.

Table 5. Data for metals and $\mathrm{NO}_{3}{ }^{-}$concentrations in $\mathrm{W}$ sector.

\begin{tabular}{cccccccccccccc}
\hline $\begin{array}{c}\text { Sector } \\
\text { Location }\end{array}$ & $\mathbf{A l}$ & $\mathbf{A s}$ & $\mathbf{B a}$ & $\mathbf{C d}$ & $\mathbf{C r}$ & $\mathbf{C o}$ & $\mathbf{C u}$ & $\mathbf{M o}$ & $\mathbf{N i}$ & $\mathbf{P b}$ & $\mathbf{V}$ & $\mathbf{Z n}$ & $\mathbf{N O}_{3}-$ \\
\hline \multirow{2}{*}{22} & 4579 & 3.06 & 87 & - & 6.3 & 4.5 & - & - & 1.98 & 26 & 11.2 & 51 & 56 \\
& $(0.7)$ & $(17)$ & $(1.1)$ & $(-)$ & $(1.0)$ & $(1.1)$ & $(-)$ & $(-)$ & $(0.4)$ & $(0.5)$ & $(1.3)$ & $(0.5)$ & $(1.3)$ \\
\hline \multirow{2}{*}{23} & 4232 & - & 88 & 0.12 & 5.9 & 3.6 & 2.8 & 0.25 & 3.44 & 32 & 10.3 & 46 & 62 \\
& $(0.7)$ & $(-)$ & $(1.1)$ & $(0.3)$ & $(0.9)$ & $(0.9)$ & $(0.3)$ & $(5.5)$ & $(0.7)$ & $(0.6)$ & $(1.2)$ & $(0.5)$ & $(1.5)$ \\
\hline \multirow{2}{*}{24} & 6371 & - & 103 & - & 10.4 & 5.3 & 13.3 & 0.34 & 5.47 & 65 & 13.2 & 78 & 139 \\
& $(1.0)$ & $(-)$ & $(1.3)$ & $(-)$ & $(1.7)$ & $(1.3)$ & $(1.4)$ & $(7.6)$ & $(1.1)$ & $(1.2)$ & $(1.5)$ & $(0.8)$ & $(3.3)$ \\
\hline \multirow{2}{*}{27} & 4977 & - & 92 & - & 6.7 & 4.8 & - & - & 1.19 & 35 & 14.8 & 55 & 122 \\
& $(0.8)$ & $(-)$ & $(1.2)$ & $(-)$ & $(1.1)$ & $(1.2)$ & $(-)$ & $(-)$ & $(0.2)$ & $(0.7)$ & $(1.7)$ & $(0.6)$ & $(2.9)$ \\
\hline \multirow{2}{*}{28} & 2908 & - & 62 & - & 4.9 & 3.2 & - & 0.17 & 0.17 & 26 & 9.3 & 43 & - \\
& $(0.5)$ & $(-)$ & $(0.8)$ & $(-)$ & $(0.8)$ & $(0.8)$ & $(-)$ & $(3.8)$ & $(0.0)$ & $(0.5)$ & $(1.0)$ & $(0.4)$ & $(-)$ \\
\hline \multirow{2}{*}{29} & 5015 & 2.09 & 91 & - & 8.5 & 4.2 & 1.6 & - & 3.54 & 51 & 11.8 & 66 & - \\
& $(0.8)$ & $(12)$ & $(1.2)$ & $(-)$ & $(1.4)$ & $(1.0)$ & $(0.2)$ & $(-)$ & $(0.7)$ & $(1.0)$ & $(1.3)$ & $(0.7)$ & $(-)$ \\
\hline \multirow{2}{*}{30} & 4570 & - & 85 & - & 6.4 & 3.7 & 4.2 & 0.24 & 3.89 & 42 & 10.5 & 56 & 156 \\
& $(0.7)$ & $(-)$ & $(1.1)$ & $(-)$ & $(1.0)$ & $(0.9)$ & $(0.4)$ & $(5.2)$ & $(0.7)$ & $(0.8)$ & $(1.2)$ & $(0.6)$ & $(3.7)$ \\
\hline
\end{tabular}


Table 5. Cont.

\begin{tabular}{cccccccccccccc}
\hline $\begin{array}{c}\text { Sector } \\
\text { Location }\end{array}$ & Al & As & Ba & $\mathbf{C d}$ & $\mathbf{C r}$ & $\mathbf{C o}$ & $\mathbf{C u}$ & $\mathbf{M o}$ & $\mathbf{N i}$ & $\mathbf{P b}$ & $\mathbf{V}$ & $\mathrm{Zn}$ & $\mathbf{N O}_{3}-$ \\
\hline \multirow{2}{*}{31} & 4549 & - & 79 & - & 6.4 & 4.0 & - & - & 3.22 & 39 & 12.11 .4 & 52 & 112 \\
& $(0.7)$ & $(-)$ & $(1.0)$ & $(-)$ & $(1.0)$ & $(1.0)$ & $(-)$ & $(-)$ & $(0.6)$ & $(0.7)$ & & $(0.5)$ & $(2.7)$ \\
\hline \multirow{2}{*}{32} & 3974 & - & 104 & - & 6.3 & 4.5 & - & - & 1.35 & 41 & 12.6 & 49 & 87 \\
& $(0.7)$ & $(-)$ & $(1.4)$ & $(-)$ & $(1.0)$ & $(1.1)$ & $(-)$ & $(-)$ & $(0.3)$ & $(0.8)$ & $(1.4)$ & $(0.5)$ & $(2.1)$ \\
\hline \multirow{2}{*}{33} & 5225 & - & 87 & - & 7.6 & 3.9 & 3.5 & 0.22 & 4.99 & 49 & 11.3 & 117 & 57 \\
& $(0.8)$ & $(-)$ & $(1.1)$ & $(-)$ & $(1.2)$ & $(1.0)$ & $(0.4)$ & $(4.8)$ & $(1.0)$ & $(0.9)$ & $(1.3)$ & $(1.2)$ & $(1.4)$ \\
\hline \multirow{2}{*}{34} & 3640 & - & 51 & 0.48 & 6.4 & 3.0 & 2.4 & 0.36 & 3.87 & 34 & 9.8 & 71 & 92 \\
& $(0.6)$ & $(-)$ & $(0.7)$ & $(1.0)$ & $(1.0)$ & $(0.7)$ & $(0.3)$ & $(8.1)$ & $(0.7)$ & $(0.7)$ & $(1.1)$ & $(0.7)$ & $(2.2)$ \\
\hline \multirow{2}{*}{35} & 4332 & - & 81 & - & 5.5 & 3.6 & - & 0.19 & 3.06 & 31 & 12.0 & 40 & 151 \\
& $(0.7)$ & $(-)$ & $(1.0)$ & $(-)$ & $(0.9)$ & $(0.9)$ & $(-)$ & $(4.2)$ & $(0.6)$ & $(0.6)$ & $(1 / 4)$ & $(0.4)$ & $(3.6)$ \\
\hline \multirow{2}{*}{36} & 4796 & - & 92 & - & 6.3 & 4.7 & 5.5 & 0.28 & 1.93 & 58 & 9.6 & 57 & 36 \\
& $(0.8)$ & $(-)$ & $(1.2)$ & $(-)$ & $(1.0)$ & $(1.1)$ & $(0.6)$ & $(6.1)$ & $(0.4)$ & $(1.1)$ & $(1.1)$ & $(0.6)$ & $(0.9)$ \\
\hline \multirow{2}{*}{37} & 5062 & - & 84 & 0.06 & 6.0 & 3.9 & 14.5 & - & 5.06 & 36 & 7.6 & 58 & 78 \\
& $(0.8)$ & $(-)$ & $(1.1)$ & $(0.1)$ & $(1.0)$ & $(1.0)$ & $(1.5)$ & $(-)$ & $(1.0)$ & $(0.7)$ & $(0.9)$ & $(0.6)$ & $(1.9)$ \\
\hline \multirow{2}{*}{38} & 5808 & - & 92 & - & 6.7 & 4.6 & 6.7 & - & 4.20 & 25 & 8.1 & 52 & 85 \\
& $(0.9)$ & $(-)$ & $(1.2)$ & $(-)$ & $(1.1)$ & $(1.1)$ & $(0.7)$ & $(-)$ & $(0.8)$ & $(0.5)$ & $(0.9)$ & $(0.5)$ & $(2.0)$ \\
\hline Mean & 4669 & 0.3 & 85 & 0.04 & 6.7 & 4.1 & 3.6 & 0.14 & 3.16 & 39 & 11 & 59 & 82 \\
STDEV & 840 & 0.9 & 14 & 0.12 & 1.3 & 0.63 & 4.7 & 0.14 & 1.55 & 12 & 1.9 & 19 & 49 \\
Max & 6371 & 3.06 & 104 & 0.48 & 10.4 & 5.3 & 14.5 & 0.36 & 5.47 & 65 & 14.8 & 117 & 156 \\
Min & 2908 & - & 51 & - & 4.9 & 3.0 & - & - & 0.17 & 25 & 7.6 & 40 & - \\
Sector Location & $\mathrm{Al}$ & $\mathrm{As}$ & $\mathrm{Ba}$ & $\mathrm{Cd}$ & $\mathrm{Cr}$ & $\mathrm{Co}$ & $\mathrm{Cu}$ & $\mathrm{Mo}$ & $\mathrm{Ni}$ & $\mathrm{Pb}$ & $\mathrm{V}$ & $\mathrm{Zn}$ & $\mathrm{NO}_{3}^{-}$ \\
\hline
\end{tabular}

Note: Values reported in $\mathrm{mg} \mathrm{kg}^{-1}$ in lichen; STDEV: standard deviation. $\pm 1 \sigma$ of background mean; - Nil; ( ) are pollutant ratios with ambient background averages.

Table 6. Data for metals and $\mathrm{NO}_{3}{ }^{-}$concentrations in $\mathrm{N}$ sector.

\begin{tabular}{|c|c|c|c|c|c|c|c|c|c|c|c|c|c|}
\hline $\begin{array}{l}\text { Sector } \\
\text { Location }\end{array}$ & Al & As & Ba & $\mathrm{Cd}$ & $\mathrm{Cr}$ & Co & $\mathrm{Cu}$ & Mo & $\mathrm{Ni}$ & $\mathbf{P b}$ & V & $\mathrm{Zn}$ & $\mathrm{NO}_{3}{ }^{-}$ \\
\hline 39 & $\begin{array}{l}4178 \\
(0.7)\end{array}$ & $\begin{array}{l}- \\
(-)\end{array}$ & $\begin{array}{c}67 \\
(0.9)\end{array}$ & (-) & $\begin{array}{c}5.0 \\
(0.8)\end{array}$ & $\begin{array}{c}3.3 \\
(0.8)\end{array}$ & $\begin{array}{l}- \\
(-)\end{array}$ & $\begin{array}{c}0.50 \\
(11.2)\end{array}$ & $\begin{array}{l}0.76 \\
(0.1)\end{array}$ & $\begin{array}{c}34 \\
(0.7)\end{array}$ & (-) & $\begin{array}{c}29 \\
(0.3)\end{array}$ & $\begin{array}{c}65 \\
(1.5)\end{array}$ \\
\hline 40 & $\begin{array}{l}4916 \\
(0.8)\end{array}$ & $(-)$ & $\begin{array}{c}68 \\
(0.9)\end{array}$ & $(-)$ & $\begin{array}{c}5.7 \\
(0.9)\end{array}$ & $\begin{array}{c}4.0 \\
(1.0)\end{array}$ & $\dot{-}$ & $\begin{array}{c}- \\
(-)\end{array}$ & $\begin{array}{l}2.73 \\
(0.5)\end{array}$ & $\begin{array}{c}46 \\
(0.9)\end{array}$ & $\begin{array}{c}- \\
(-)\end{array}$ & $\begin{array}{c}44 \\
(0.5)\end{array}$ & $\begin{array}{c}182 \\
(4.3)\end{array}$ \\
\hline 41 & $\begin{array}{l}1459 \\
(0.2)\end{array}$ & $\begin{array}{c}- \\
(-)\end{array}$ & $\begin{array}{c}49 \\
(0.6)\end{array}$ & $(-)$ & $\begin{array}{c}1.9 \\
(0.3)\end{array}$ & $\begin{array}{c}1.5 \\
(0.4)\end{array}$ & $\overline{-}$ & $(-)$ & $(-)$ & $\begin{array}{l}6.67 \\
(0.1)\end{array}$ & $\begin{array}{c}- \\
(-)\end{array}$ & $\begin{array}{l}10.2 \\
(0.1)\end{array}$ & $\begin{array}{l}101 \\
(2.4)\end{array}$ \\
\hline 42 & $\begin{array}{l}1123 \\
(0.2)\end{array}$ & $\begin{array}{l}- \\
(-)\end{array}$ & $\begin{array}{c}22 \\
(0.3)\end{array}$ & $(-$ & $\begin{array}{c}2.4 \\
(0.4)\end{array}$ & $\begin{array}{c}2 \\
(0.5)\end{array}$ & $\begin{array}{l}- \\
(-)\end{array}$ & $\begin{array}{l}- \\
(-)\end{array}$ & $\begin{array}{l}- \\
(-)\end{array}$ & $\begin{array}{l}9.12 \\
(0.2)\end{array}$ & $\begin{array}{l}- \\
(-)\end{array}$ & $\begin{array}{c}5.8 \\
(0.1)\end{array}$ & $\begin{array}{c}251 \\
(6.0)\end{array}$ \\
\hline 43 & $\begin{array}{l}4077 \\
(0.6)\end{array}$ & $(-)$ & $\begin{array}{c}92 \\
(1.2)\end{array}$ & (-) & $\begin{array}{c}5.3 \\
(0.9)\end{array}$ & $\begin{array}{c}4.3 \\
(1.1)\end{array}$ & (-) & $\begin{array}{l}- \\
(-)\end{array}$ & $\begin{array}{c}1.3 \\
(0.3)\end{array}$ & $\begin{array}{c}43 \\
(0.8)\end{array}$ & $\begin{array}{c}6.3 \\
(0.7)\end{array}$ & $\begin{array}{c}41 \\
(0.4)\end{array}$ & $\begin{array}{c}82 \\
(2.0)\end{array}$ \\
\hline 44 & $\begin{array}{l}3908 \\
(0.6)\end{array}$ & $\begin{array}{c}- \\
(-)\end{array}$ & $\begin{array}{c}64 \\
(0.8)\end{array}$ & $\begin{array}{c}- \\
(-)\end{array}$ & $\begin{array}{c}6.5 \\
(1.0)\end{array}$ & $\begin{array}{c}3.5 \\
(0.9)\end{array}$ & $\begin{array}{c}- \\
(-)\end{array}$ & $\begin{array}{c}- \\
(-)\end{array}$ & $\begin{array}{c}- \\
(-)\end{array}$ & $\begin{array}{c}37 \\
(0.7)\end{array}$ & $\begin{array}{l}19.4 \\
(2.2)\end{array}$ & $\begin{array}{c}35 \\
(0.4)\end{array}$ & $\begin{array}{l}339 \\
(8.1)\end{array}$ \\
\hline 45 & $\begin{array}{l}3067 \\
(0.5)\end{array}$ & $(-$ & $\begin{array}{c}59 \\
(0.8)\end{array}$ & $\begin{array}{c}- \\
(-)\end{array}$ & $\begin{array}{c}4.6 \\
(0.7)\end{array}$ & $\begin{array}{c}2.8 \\
(0.7)\end{array}$ & $\begin{array}{c}- \\
(-)\end{array}$ & $\begin{array}{l}0.20 \\
(4.4)\end{array}$ & $\begin{array}{c}1.8 \\
(0.3)\end{array}$ & $\begin{array}{c}20 \\
(0.4)\end{array}$ & $\begin{array}{c}3.0 \\
(0.3)\end{array}$ & $\begin{array}{c}40 \\
(0.4)\end{array}$ & $\begin{array}{l}304 \\
(7.2)\end{array}$ \\
\hline 46 & $\begin{array}{l}2184 \\
(0.3)\end{array}$ & $\begin{array}{c}1.9 \\
(11)\end{array}$ & $\begin{array}{c}56 \\
(0.7)\end{array}$ & $(-)$ & $\begin{array}{c}3.5 \\
(0.6)\end{array}$ & $\begin{array}{c}1.7 \\
(0.4)\end{array}$ & $\overline{(-}$ & $\overline{(-}$ & $\begin{array}{c}0.2 \\
(0.0)\end{array}$ & $\begin{array}{c}24 \\
(0.5)\end{array}$ & $\begin{array}{c}7.5 \\
(0.8)\end{array}$ & $\begin{array}{c}40 \\
(0.4)\end{array}$ & $\begin{array}{c}78 \\
(1.9)\end{array}$ \\
\hline 47 & $\begin{array}{l}3177 \\
(0.5)\end{array}$ & $(-)$ & $\begin{array}{c}44 \\
(0.6)\end{array}$ & $(-)$ & $\begin{array}{c}4.4 \\
(0.7)\end{array}$ & $\begin{array}{c}2.2 \\
(0.5)\end{array}$ & $\begin{array}{c}1.6 \\
(0.2)\end{array}$ & $\begin{array}{l}0.70 \\
(16)\end{array}$ & $\begin{array}{c}2.9 \\
(0.6)\end{array}$ & $\begin{array}{c}23 \\
(0.4)\end{array}$ & $\begin{array}{c}9.3 \\
(1.0)\end{array}$ & $\begin{array}{c}30 \\
(0.3)\end{array}$ & $\begin{array}{c}56 \\
(1.3)\end{array}$ \\
\hline 48 & $\begin{array}{l}4592 \\
(0.7)\end{array}$ & $\begin{array}{l}- \\
(-)\end{array}$ & $\begin{array}{c}78 \\
(1.0)\end{array}$ & $\begin{array}{l}0.23 \\
(0.5)\end{array}$ & $\begin{array}{c}5.6 \\
(0.9)\end{array}$ & $\begin{array}{c}3.0 \\
(0.7)\end{array}$ & $\begin{array}{c}6.4 \\
(0.7)\end{array}$ & $\begin{array}{l}- \\
(-)\end{array}$ & $\begin{array}{c}4.4 \\
(0.8)\end{array}$ & $\begin{array}{l}19.5 \\
(0.4)\end{array}$ & $\begin{array}{c}6.3 \\
(0.7)\end{array}$ & $\begin{array}{c}31 \\
(0.3)\end{array}$ & $\begin{array}{c}564 \\
(13.4)\end{array}$ \\
\hline 49 & $\begin{array}{l}3380 \\
(0.5)\end{array}$ & $\begin{array}{l}- \\
(-)\end{array}$ & $\begin{array}{c}77 \\
(1.0)\end{array}$ & $\begin{array}{l}- \\
(-)\end{array}$ & $\begin{array}{c}6.2 \\
(1.0)\end{array}$ & $\begin{array}{c}2.6 \\
(0.6)\end{array}$ & $\begin{array}{l}- \\
(-)\end{array}$ & $\begin{array}{l}- \\
(-)\end{array}$ & $\begin{array}{c}1.3 \\
(0.3)\end{array}$ & $\begin{array}{c}42 \\
(0.8)\end{array}$ & $\begin{array}{c}9.2 \\
(1.0)\end{array}$ & $\begin{array}{c}31 \\
(0.3)\end{array}$ & $\begin{array}{c}25 \\
(0.6)\end{array}$ \\
\hline 50 & $\begin{array}{l}4352 \\
(0.7)\end{array}$ & $\begin{array}{c}- \\
(-)\end{array}$ & $\begin{array}{c}77 \\
(1.0)\end{array}$ & $\begin{array}{l}0.40 \\
(0.8)\end{array}$ & $\begin{array}{c}5.4 \\
(0.9)\end{array}$ & $\begin{array}{c}2.7 \\
(0.7)\end{array}$ & $\begin{array}{c}9.4 \\
(1.0)\end{array}$ & $\begin{array}{c}- \\
(-)\end{array}$ & $\begin{array}{c}5.0 \\
(1.0)\end{array}$ & $\begin{array}{l}16.7 \\
(0.3)\end{array}$ & $\begin{array}{c}6.7 \\
(0.8)\end{array}$ & $\begin{array}{c}30 \\
(0.3)\end{array}$ & $\begin{array}{c}426 \\
(10.2)\end{array}$ \\
\hline
\end{tabular}


Table 6. Cont.

\begin{tabular}{cccccccccccccc}
\hline $\begin{array}{c}\text { Sector } \\
\text { Location }\end{array}$ & $\mathbf{A l}$ & $\mathbf{A s}$ & $\mathbf{B a}$ & $\mathbf{C d}$ & $\mathbf{C r}$ & $\mathbf{C o}$ & $\mathbf{C u}$ & $\mathbf{M o}$ & $\mathbf{N i}$ & $\mathbf{P b}$ & $\mathbf{V}$ & $\mathbf{Z n}$ & $\mathbf{N O}_{3}{ }^{-}$ \\
\hline \multirow{2}{*}{51} & 1714 & - & 114 & - & 3.0 & 3.0 & - & - & - & 35 & 16.7 & 27 & 351 \\
& $(0.3)$ & $(-)$ & $(1.5)$ & $(-)$ & $(0.5)$ & $(0.7)$ & $(-)$ & $(-)$ & $(-)$ & $(0.7)$ & $(1.9)$ & $(0.3)$ & $(8.4)$ \\
\hline \multirow{2}{*}{52} & 4368 & - & 84 & - & 6.4 & 3.7 & - & - & 2.4 & 24 & 8.1 & 36 & 23 \\
& $(0.7)$ & $(-)$ & $(1.1)$ & $(-)$ & $(1.0)$ & $(0.9)$ & $(-)$ & $(-)$ & $(0.5)$ & $(0.5)$ & $(0.9)$ & $(0.4)$ & $(0.5)$ \\
\hline \multirow{2}{*}{53} & 5195 & - & 78 & 0.20 & 5.2 & 2.2 & 9.7 & - & 3.8 & 8.9 & 6.5 & 28 & 14 \\
& $(0.8)$ & $(-)$ & $(1.0)$ & $(0.4)$ & $(0.8)$ & $(0.5)$ & $(1.0)$ & $(-)$ & $(0.7)$ & $(0.2)$ & $(0.7)$ & $(0.3)$ & $(0.3)$ \\
\hline \multirow{2}{*}{54} & 5589 & - & 95 & 0.30 & 6.1 & 3.0 & 11.4 & - & 5.4 & 15.3 & 6.6 & 35 & 83 \\
& $(0.9)$ & $(-)$ & $(1.2)$ & $(0.6)$ & $(1.0)$ & $(0.7)$ & $(1.2)$ & $(-)$ & $(1.0)$ & $(0.3)$ & $(0.7)$ & $(0.4)$ & $(2.0)$ \\
\hline \multirow{2}{*}{55} & 6045 & - & 119 & 1.40 & 8.6 & 5.7 & 8.3 & - & 4.8 & 139 & 19 & 277 & 204 \\
& $(1.0)$ & $(-)$ & $(1.5)$ & $(2.9)$ & $(1.4)$ & $(1.4)$ & $(0.9)$ & $(-)$ & $(0.9)$ & $(2.7)$ & $(2.1)$ & $(2.9)$ & $(4.9)$ \\
\hline Mean & 3725 & 0.11 & 73 & 0.15 & 5.0 & 3.0 & 2.8 & 0.082 & 2.2 & 32 & 7.8 & 45 & 185 \\
STDEV & 1448 & 0.46 & 24 & 0.35 & 1.7 & 1.0 & 4.3 & 0.20 & 2.0 & 30 & 5.9 & 60 & 163 \\
Max & 6045 & 1.9 & 119 & 1.4 & 8.6 & 5.7 & 11.4 & 0.70 & 5.4 & 139 & 19.4 & 277 & 564 \\
Min & 1123 & - & 22 & - & 1.9 & 1.5 & - & - & - & 6.67 & - & 5.8 & 14 \\
\hline
\end{tabular}

Note: Values reported in $\mathrm{mg} \mathrm{kg}^{-1}$ in lichen; STDEV: standard deviation. $\pm 1 \sigma$ of background mean; - Nil; ( ) are pollutant ratios with ambient background averages.

Trace elements and $\mathrm{NO}_{3}{ }^{-}$were detected at all data points with the exception of $\mathrm{As}, \mathrm{Cd}, \mathrm{Mo}$ and $\mathrm{Cu}$. Arsenic was detected at $23 \%$ of the locations ranging between less than 0.025 and $11.2 \mathrm{mg} \mathrm{kg}^{-1}$ with a mean value of $0.6 \mathrm{mg} \mathrm{kg}^{-1} ; \mathrm{Cd}$ and Mo were slightly higher than As with $46.9 \%$ of locations containing between 0.06 and $0.48 \mathrm{mg} \mathrm{kg}^{-1 ;} 39 \%$ of locations contained Mo ranging between 0.06 and $1.02 \mathrm{mg} \mathrm{kg}^{-1}$. Finally, $\mathrm{Cu}$ was detected at $71 \%$ of locations and ranged between 1.6 and $89 \mathrm{mg} \mathrm{kg}^{-1}$.

Some metals ( $\mathrm{Al}, \mathrm{Ba}, \mathrm{Mo}, \mathrm{Zn}$ ) detected across the valley are ubiquitously distributed, suggesting little to no enhancement while others ( $\mathrm{As}, \mathrm{Co}, \mathrm{Cr}, \mathrm{Cu}, \mathrm{Ni}, \mathrm{Pb}, \mathrm{V}$ ) are higher in the $\mathrm{E}$ and $\mathrm{S}$ sectors of the valley with the $S$ sector containing the Interstate 15 (I-15) corridor (Figure 2; Tables 3-6). Although the $\mathrm{S}$ sector contained elevated metals along the I-15 corridor, many were detected more than $0.25 \mathrm{~km}$ from the highway. While those samples collected within $20 \mathrm{~m}$ of the highway may contained pollution from direct vehicle traffic, it is not seen as a major contributor to the overall data set. According to Botsou et al. [30] and others for instance, detections of metals near roads and highways are not an issue of concern as vehicle emissions rarely excessed $10 \mathrm{~m}$ from the road traffic [31,32].

A dissimilar distribution was observed for $\mathrm{NO}_{3}{ }^{-}$as observed with metals; highest in the $\mathrm{N}$ sector ranging between 14 and $564 \mathrm{mg} \mathrm{kg}^{-1}$ with locations 48, 50, and $51 \geq 1 \sigma\left(564,426,351 \mathrm{mg} \mathrm{kg}^{-1}\right.$, respectively). The $\mathrm{W}$ sector ranged between non-detect (ND) and $156 \mathrm{mg} \mathrm{kg}^{-1}$ with locations 24,30 and $35 \geq 1 \sigma\left(139,156\right.$ and $151 \mathrm{mg} \mathrm{kg}^{-1}$, respectively) with the lowest $\mathrm{NO}_{3}{ }^{-}$detected in the $\mathrm{S}$ and $\mathrm{E}$ sectors. $\mathrm{NO}_{3}{ }^{-}$ranged between $\mathrm{ND}$ and $129 \mathrm{mg} \mathrm{kg}^{-1}$ in the $\mathrm{S}$ sector with locations 13 and 15 containing $\geq 1 \sigma\left(102,129 \mathrm{mg} \mathrm{kg}^{-1}\right.$, respectively) while $\mathrm{NO}_{3}{ }^{-}$ranged between ND and 53 in the E sector with locations 57 and $61 \geq 1 \sigma\left(53,50 \mathrm{mg} \mathrm{kg}{ }^{-1}\right.$, respectively). $\mathrm{NO}_{3}{ }^{-}$are greater than $1.5 \times$ background averages with 13 locations in the $\mathrm{N}$ sector (loci 40-45, 48,50-51,54-55) and 11 locations in the $\mathrm{W}$ sector (loci 23-24, 27, 30-35, 37-38). The $\mathrm{S}$ sector contained 6 locations $>1.5 \times$ background $\mathrm{NO}_{3}{ }^{-}$(loci 7, 9, 11, $13,15-16)$ with no locations in the E. It is important to point out that the sectors containing the highest $\mathrm{NO}_{3}{ }^{-}$had the lowest metals and conversely, sectors with the lowest $\mathrm{NO}_{3}{ }^{-}$contained the highest metals concentrations.

To better understand observed trace elements ("metals") and $\mathrm{NO}_{3}{ }^{-}$patterns, principal component analysis (PCA) was performed on the 4 sectors of Las Vegas (N, E, S, and W). Results show 2 strong associations ("clusters") in Figures 3 and 4 with cluster 1 containing more data points from the $N$ sectors while cluster 2 contains more data points from the W sector. It is significant to note that there is a minor correlation within or between the $\mathrm{S}$ and $\mathrm{E}$ sectors. Clusters 1 and 2 for example, show metals clustering in the $\mathrm{N}$ and $\mathrm{W}$ sectors of the valley where metals are low while $\mathrm{NO}_{3}{ }^{-}$was highest. Furthermore, there is overlapping correlations between clusters 1 and 2, this is indicative of similarities 
between those components and localities (Figure 3). Data for the $\mathrm{S}$ and E sectors show insignificant correlations among metals within those two sectors. While metals in the $\mathrm{N}$ and $\mathrm{W}$ were relatively similar in those two sectors, metals in the $S$ and $E$ sectors, though higher than detected in the $W$ and $N$ sectors, varied when compared within those two sectors.

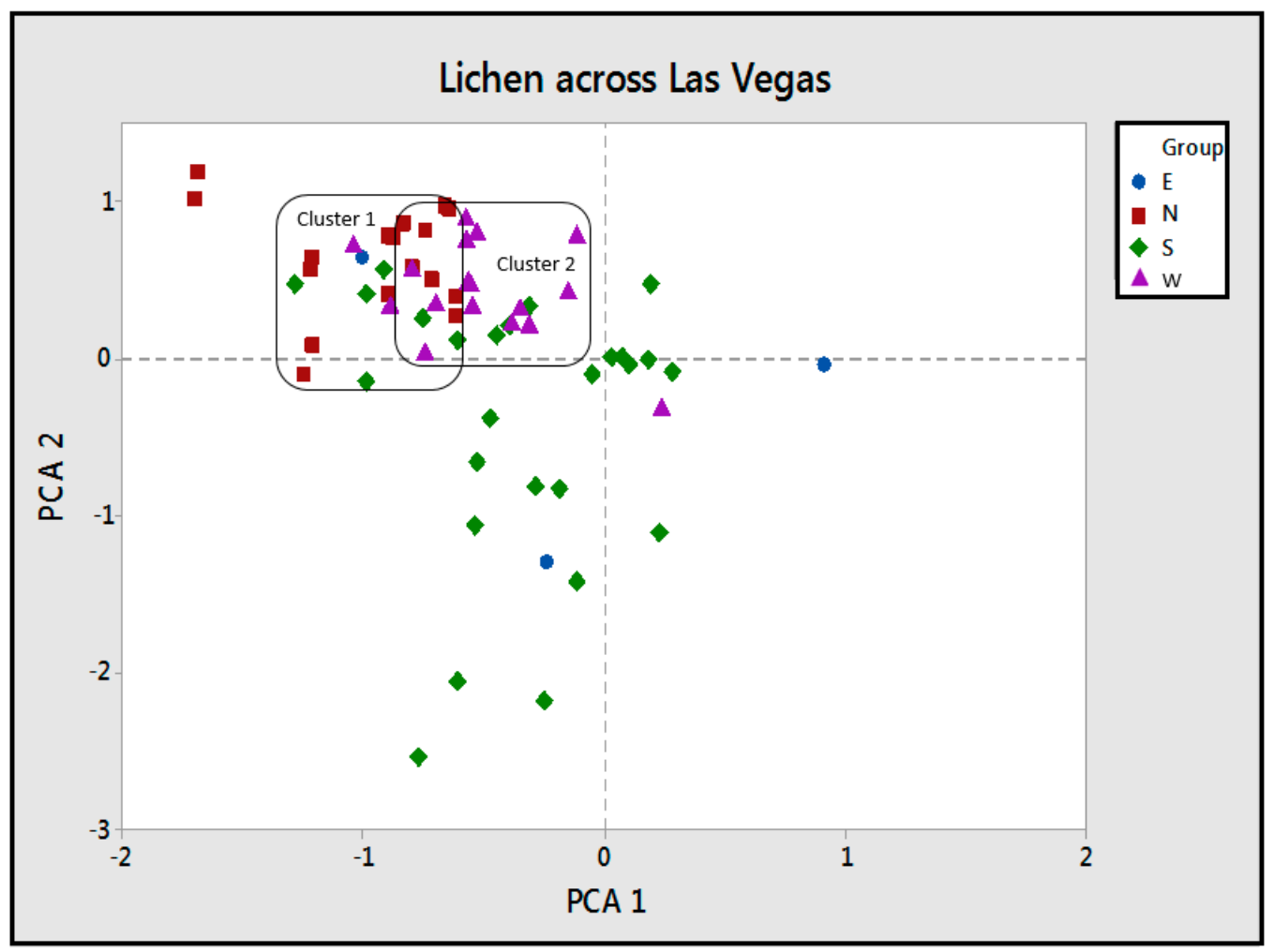

Figure 3. Principal component analysis showing two clusters across the Las Vegas Valley.

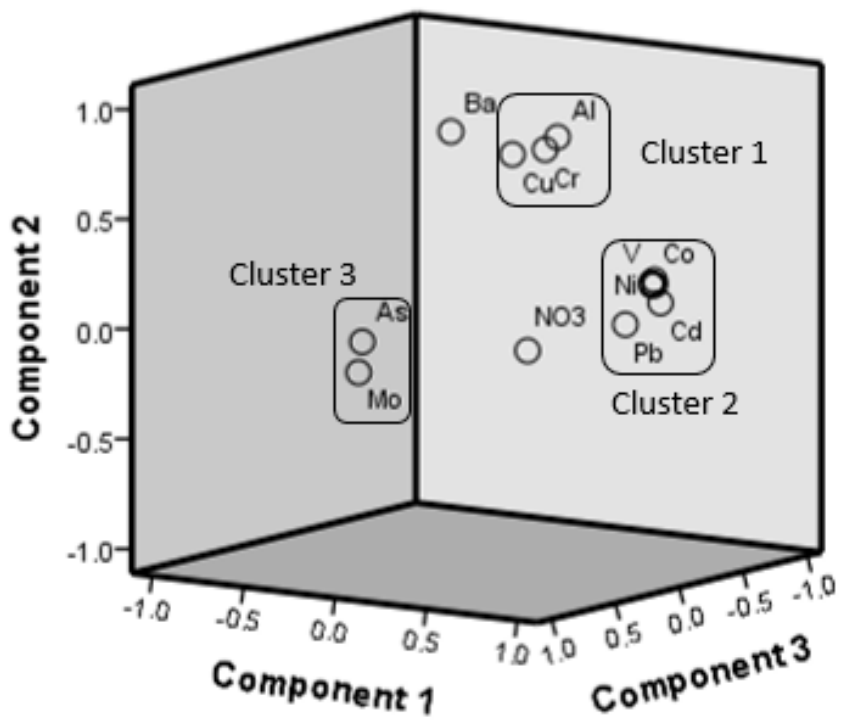

Figure 4. Component plot in rotated space explaining dataset from across the Las Vegas Valley.

With PCA showing two correlative clusters in the N and W sectors (Clusters 1 and 2; Figure 3), additional analysis was required to further describe the distribution within sectors; therefore, three (3) composite factors were assigned to further clarify metal variability in the dataset (Figure 4). Results 
show 3 groupings (clusters 1, 2 and 3) with two unassociated pollutants; $\mathrm{Ba}$ and $\mathrm{NO}_{3}{ }^{-}$. There is a strong correlation between $\mathrm{Cd}-\mathrm{Co}-\mathrm{Ni}-\mathrm{Pb}-\mathrm{V}$ as shown in cluster 1 (52.1\% of Variance); a strong correlation between $\mathrm{Cu}-\mathrm{Cr}-\mathrm{Al}$ in cluster 2 (16.1\% of Variance); and cluster 3 (12.6\% of Variance) reveals a strong correlation between As-Mo. Clusters 1, 2 and 3 are frequently detected where $\mathrm{NO}_{3}{ }^{-}$values are low, as given by their inverse correlation. Metals and $\mathrm{NO}_{3}{ }^{-}$appear enhanced when compared to background concentrations in the Las Vegas valley however, elevated metals appear to correlate with low $\mathrm{NO}_{3}{ }^{-}$in lichen biomass, finding similar to a report published in Airnow [33]. Finally, PCA clustering illustrates a west-east and north-south gradient (low-high) for pollution.

Data was run through discriminant function analysis (DFA) to further evaluate associations between pollutants in relation to elevation by evaluating data centroids. The structure matrix from DFA shows the influence of each chemical parameter on site separation, as given by their correlation with any of the discriminant functions. Results illustrate that the centroid for E, and somewhat $S$, sectors along with background (BG) are different from the $\mathrm{N}$ and $\mathrm{W}$ sectors based on chemistry and altitude. Figure 5 and Table 7 showed DF1 (altitude) when compared with DF2 (chemistry) is able to distinguish data centroids from the other sectors. DF2 appears to be based on $\mathrm{Al}, \mathrm{Cu}$ and $\mathrm{Cr}$ abundances. Overall, DFA analysis shows data centroids for the E and S sectors are dissimilar to the $\mathrm{N}$ and $\mathrm{W}$ sectors and, BG is dissimilar from all four sectors.

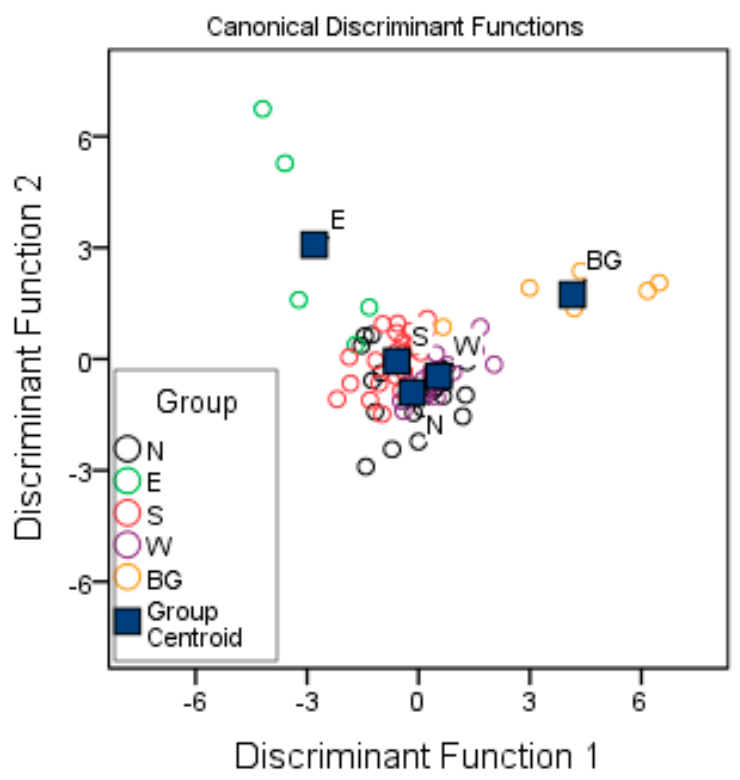

Figure 5. Discriminant Function Analysis showing the separation of sites based on sample chemical analysis.

To further describe the atmospheric dispersal of metals and $\mathrm{NO}_{3}{ }^{-}$, samples within the four sectors (N, E, S, W) were compared with background sample ratios (Tables 2-6). Data show E and S sector ratios (i.e., $\mathrm{Al}, \mathrm{As}, \mathrm{Cd}, \mathrm{Cu}, \mathrm{Ni}, \mathrm{Pb}, \mathrm{Zn}$ ) differ from the $\mathrm{N}$ and $\mathrm{W}$ sectors. Values in $\mathrm{N}$ and $\mathrm{W}$ sectors were largely less than 1.0, whereas $\mathrm{E}$ and $\mathrm{S}$ sectors ratios were principally greater than 1.0. A few metal ratios (i.e., $\mathrm{Ba}, \mathrm{Cr}, \mathrm{Co}, \mathrm{V}$ ) were greater than 1.0 consistently across the $\mathrm{S}, \mathrm{W}$ and $\mathrm{E}$ sectors while less than 1.0 in the $\mathrm{N}$ sector. Moreover, the $\mathrm{N}$ and $\mathrm{W}$ sectors show significantly lower ratios than the $\mathrm{E}$ and $\mathrm{S}$, indicative of very small inputs of air pollutants to lichen biomass. Largely, $\mathrm{NO}_{3}{ }^{-}$was shown to consistently have an inverse correlation to metals across the valley when evaluating general ratios. $\mathrm{NO}_{3}{ }^{-}$was consistently greater than 1.0 in the $\mathrm{N}$ and $\mathrm{W}$ sectors where metal (i.e., $\mathrm{As}, \mathrm{Cr}, \mathrm{Cu}, \mathrm{Ni}, \mathrm{Pb}$ ) ratios were less than 1.0 and less than $1.0\left(\mathrm{NO}_{3}{ }^{-}\right)$where metal ratios were constantly greater than 1.0. Finally, ratio data show $\mathrm{NO}_{3}{ }^{-}$and metals follow PCA and DFA interpretations (west-east and north-south gradient) with $\mathrm{NO}_{3}{ }^{-}$high where metals are low. 
Table 7. Structure matrix showing influence of each chemical parameter on site separation.

\begin{tabular}{ccccc}
\hline \multicolumn{5}{c}{ Function } \\
\hline Elevation $(\mathrm{m})$ & 1 & 2 & 3 & 4 \\
$\mathrm{Al}$ & $0.717^{*}$ & 0.142 & 0.070 & -0.097 \\
$\mathrm{Cu}$ & -0.236 & $0.593^{*}$ & 0.304 & -0.188 \\
$\mathrm{Cr}$ & -0.217 & $0.572^{*}$ & 0.038 & 0.91 \\
$\mathrm{NO}_{3}$ & -0.256 & $0.537^{*}$ & 0.247 & -0.248 \\
$\mathrm{Mo}$ & 0.024 & -0.383 & $0.561^{*}$ & 0.142 \\
$\mathrm{Ba}$ & -0.046 & -0.122 & $-0.405^{*}$ & 0.037 \\
$\mathrm{As}$ & -0.209 & 0.205 & $-0.373^{*}$ & 0.178 \\
$\mathrm{Cd}$ & -0.066 & 0.004 & $-0.333^{*}$ & 0.124 \\
$\mathrm{Zn}$ & -0.044 & 0.145 & $0.287^{*}$ & 0.162 \\
$\mathrm{~V}$ & -0.010 & 0.182 & $0.256^{*}$ & -0.005 \\
$\mathrm{Ni}$ & -0.109 & 0.136 & $0.240^{*}$ & -0.033 \\
$\mathrm{Co}$ & -0.107 & 0.159 & $0.200^{*}$ & 0.089 \\
$\mathrm{~Pb}$ & -0.100 & 0.132 & $0.182^{*}$ & 0.023 \\
\hline
\end{tabular}

Note: Pooled within-groups correlations between discriminating variables and standardized canonical discriminant functions. Variables ordered by absolute size of correlation within function. Largest absolute correlation between each variable and any discriminant function. Coefficients $>0.05$ are marked by “*”.

There is a direct correlation between specific metal ratios (i.e., $\mathrm{Cu} / \mathrm{Pb}, \mathrm{Co} / \mathrm{Cu}$, and $\mathrm{Cr} / \mathrm{Pb}$ ) and anthropogenic enhancement according to Font et al. [28]. With their research in mind, it was important to assess known associations of $\mathrm{Cu} / \mathrm{Pb}, \mathrm{Co} / \mathrm{Cu}$, and $\mathrm{Cr} / \mathrm{Pb}$ within $\pm 1 \sigma$ of background conditions at a $95 \%$ confidence level (CI). Metal ratios in the $\mathrm{S}$ and $\mathrm{E}$ sectors were within $\mathrm{CI}$ of background mean data with $67 \%$ and $62 \% \mathrm{Cu} / \mathrm{Pb} ; 67 \%$ and $77 \% \mathrm{Cr} / \mathrm{Pb}$; and $90 \%$ and $92 \% \mathrm{Co} / \mathrm{Cu}$ at $\pm 1 \sigma$ of background (Table 8). Although $\mathrm{Cu} / \mathrm{Pb}$ and $\mathrm{Co} / \mathrm{Cu}$ ratios were within $\mathrm{CI}, \mathrm{Cr} / \mathrm{Pb}$ ratios were marginally outside $\mathrm{CI}$ in the $\mathrm{S}$ and $\mathrm{E}$ sectors $(67 \%$ vs. $77 \%)$, whereas in the $\mathrm{N}$ and $\mathrm{W}$ sectors, ratios were outside the $\mathrm{CI}$ consistently for $\mathrm{Cu} / \mathrm{Pb}, \mathrm{Cr} / \mathrm{Pb}$, and $\mathrm{Co} / \mathrm{Cu}$. Findings are similar with PCA data showing two clusters (S and E) within the 95\% CI. Data are consistent when compared with the distribution patterns illustrated with ratios and concentrations data for metals and $\mathrm{NO}_{3}{ }^{-}$across the four sectors $(\mathrm{N}, \mathrm{E}, \mathrm{S}, \mathrm{W})$ with the $\mathrm{E}$ and $\mathrm{S}$ sectors containing higher values than the $\mathrm{N}$ and $\mathrm{W}$ sectors.

Table 8. Selected metal ratios for S, E, N and W sectors.

\begin{tabular}{cccc}
\hline Location/data & $\mathbf{C u} / \mathbf{P b}$ & $\mathbf{C r} / \mathbf{P b}$ & $\mathbf{C o} / \mathbf{C u}$ \\
\hline Sector means & $0.291 / 0.387 / 0.476 / 0.147$ & $0.174 / 0.286 / 0.225 / 0.178$ & $0.550 / 0.684 / 0.740 / 1.005$ \\
STDEV & $0.174 / 0.309 / 0.404 / 0.120$ & $0.127 / 0.247 / 0.129 / 0.037$ & $0.438 / 0.594 / 0.767 / 0.725$ \\
$\% \pm 1 \sigma$ & $67 / 62 / 24 / 20$ & $67 / 77 / 76 / 93$ & $90 / 92 / 47 / 53$ \\
\hline
\end{tabular}

Note: Data are presented in order of S/E/ N/W sectors. Percentage is within $1 \sigma$ of background mean within $95 \%$ CI. STDEV: standard deviation. $\pm 1 \sigma$ of background mean. All data is available in supplementary tables.

The $\mathrm{N}$ and $\mathrm{W}$ sector ratios (i.e., $24,20 \mathrm{Cu} / \mathrm{Pb}, 76,93 \mathrm{Cr} / \mathrm{Pb}$ and $47,53 \mathrm{Co} / \mathrm{Cu}$ ) exhibited no obvious

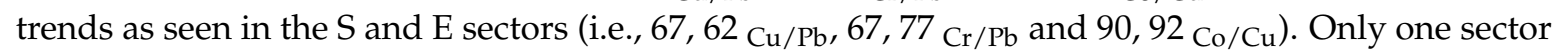
showed a single corresponding metal ratio for $\mathrm{Cr} / \mathrm{Pb}$ within $1 \sigma$ of background $\mathrm{CI}$ : the $\mathrm{N}$ sector at $76 \%$ of background, a value similar to the $\mathrm{S}$ and $\mathrm{E}$ sectors. Although the $\mathrm{N}$ sector $\mathrm{Cr} / \mathrm{Pb}$ was within the predictive model, the remaining $\mathrm{N}$ and $\mathrm{W}$ sector ratios were outside the $\mathrm{CI}$ and therefore, this single observed data point $(76 \%)$ is likely an outlier of no significant value. Finally, data indicate that metals in the $\mathrm{S}$ and $\mathrm{E}$ sectors are enriched and associated with dissimilar influences observed in the $\mathrm{N}$ and W sectors.

Windrose patterns were collected from five air monitoring stations across the Las Vegas valley and only used for comparative analysis with regards to pollution detected in lichen biomass. These stations are monitored by the USEPA all year for wind direction (Figure 6) with the stations near the 
center of each of the four sectors $(\mathrm{N}, \mathrm{E}, \mathrm{S}, \mathrm{W})$. Charting of wind data shows a pattern of winds entering the Las Vegas valley typically from the south-southwest (USEPA 32-3-1019), minor winds entering from the northwest USEPA 32-3-75), with the general direction trending south to north-northeast. Once in the valley, winds move from the south and easterly across Las Vegas, mixing and swirling in the central portion of the Valley before exiting on the north and south sides of Frenchman Mountain.

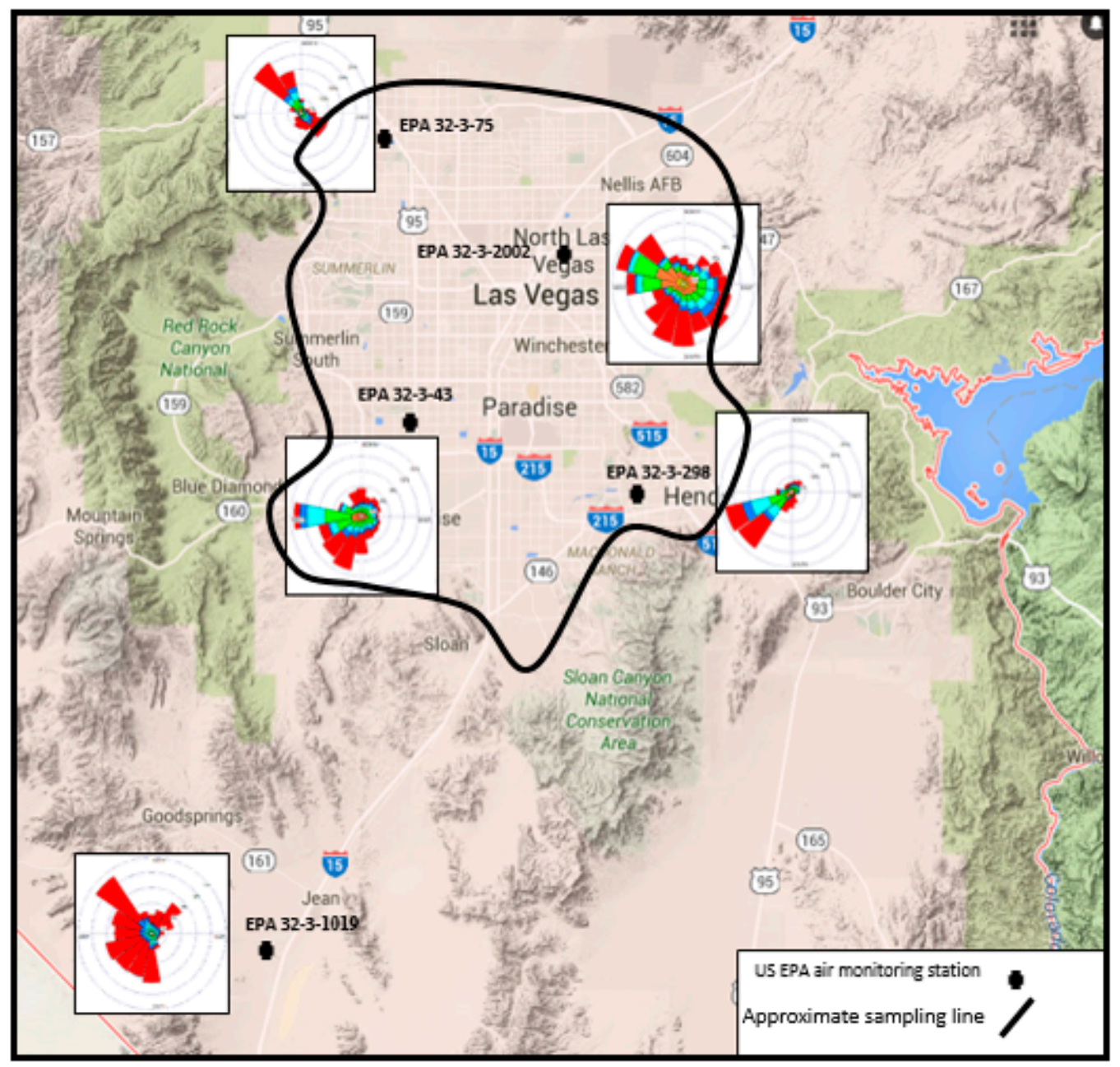

Figure 6. Wind direction with Windrose patterns from across the Las Vegas Valley (Google maps).

Wind data were analogous to analytical results in the W, N, E and S sectors. Lichen data was predictable in the $\mathrm{W}, \mathrm{N}$ and $\mathrm{E}$ sectors with regards to pollution concentrations in lichen biomass however, results from the $\mathrm{S}$ sector contained elevated metals and low $\mathrm{NO}_{3}{ }^{-}$. This outcome of the $\mathrm{S}$ sector containing elevated metals in lichen biomass does not follow windrose diagrams. Wind patterns within the valley would suggest that the $S$ sectors should have lower metals than the $W, N$, and E sectors as there is no major traffic (vehicle) patterns or industry other than the I-15 corridor and therefore, one would expect that metal concentrations to be lower. It is possible that elevated metals in the $\mathrm{S}$ sector are the result of external sources; i.e., an alternate population center. Another noteworthy trend observed was $\mathrm{NO}_{3}$; it was within $1 \sigma$ of background conditions $57 \%$ of the time across the valley; however, the $\mathrm{S}$ sector data are similar to the E sector where $\mathrm{NO}_{3}{ }^{-}$was within $1 \sigma$ of background conditions $100 \%$ of the time. Nitrate data for the $\mathrm{N}$ and $\mathrm{W}$ sectors were $<33 \%$ of $1 \sigma$ of background concentrations. 


\section{Discussion}

Studies have used lichens for documenting metal deposition across the world as it is well known that lichen absorbs metals and nitrogen [2,16,34-36]. Researchers have evaluated the distribution of contaminants in relation to wind patterns in large population centers like Los Angeles as well as industrial areas $[3,37]$. Studies have shown that metal contamination from anthropogenic sources originate from $\mathrm{PM}_{2.5}$ and $\mathrm{PM}_{10}$ atmospheric input [3,16]. Moreover, Lodenius et al. [38] established correlations between $\mathrm{PM}_{2.5}$ and $\mathrm{PM}_{10}$ air pollution in association with lichens near urban centers regardless of distance from highways [32]. Finally, lichens as a bio-indicator for pollution have never been studied within the Las Vegas valley.

Findings identified two areas of elevated metal contamination, those areas are the $S$ and E sectors with BG levels dissimilar from the four sectors (Figure 2). Metal clustering in the E sector of the Las Vegas Valley indicates pollution is following established wind patterns. Data show a clustering of elevated metals in the $S$ sector as well, an area with minimal urbanization or industry. Although the $S$ sector has the I-15 corridor traversing through its center, it is not seen as a major contributor as other researchers have documented vehicle emissions rarely exceed beyond $10 \mathrm{~m}$ from the source; i.e., the highway [31,32]. It is possible that elevated metals in the $S$ sector result from pollution migrating into the valley, based on wind patterns (Figure 6). Migrating pollution from outside sources is not new as Demiray et al. [39] identified elevated metals in lichen biomass downwind from a population center; pollution originating from an outside source, not the urban area studied. Their findings, along with others, identified higher concentrations of metals (e.g., $\mathrm{Cu}, \mathrm{Ni}, \mathrm{Pb}, \mathrm{V}$ ) originating from areas upwind from study area [37]. The $S$ sector for instance is meteorologically upwind from the population and industrial areas of Las Vegas and therefore, should have lower levels of target pollution.

Principal component analysis (PCA) identified 2 strong correlations; clusters 1 and 2 display metals clustering in the $\mathrm{N}$ and $\mathrm{W}$ sectors of the valley where metals were low while $\mathrm{NO}_{3}{ }^{-}$was highest. Data also showed $S$ and E sectors with minor to no correlations among metals. Ungureanu et al. [40] also used multivariate statistical analysis to show vehicle emissions ("anthropogenic enhancement") represent the most important sources of $\mathrm{Cd}-\mathrm{Co}-\mathrm{Ni}-\mathrm{Pb}-\mathrm{V}$ and $\mathrm{Cu}-\mathrm{Cr}-\mathrm{Al}$ in an urban environment. These finding show a similar relationship with metals as published by Ungureanu et al. [40] in similar urban areas. Furthermore, DFA data showed (chemistry ( $\mathrm{Al}, \mathrm{Cu}, \mathrm{Cr}$ ) and altitude) that the $\mathrm{E}$ and $\mathrm{S}$ sector data centroids, along with BG, are different from the $\mathrm{N}$ and $\mathrm{W}$ sectors. This difference suggest that the $\mathrm{E}$ and $\mathrm{S}$ sectors are enhanced by anthropogenic activities while the $\mathrm{N}$ and $\mathrm{W}$ sectors have less enhancement. Data for PCA and DFA correlates with elemental concentrations and metal ratios across the valley.

Metal ratios show a distinct pattern in the $\mathrm{S}$ and $\mathrm{E}$ sectors were background mean $\mathrm{CI}(95 \%)$ data is within $67 \%$ and $62 \% \mathrm{Cu} / \mathrm{Pb} ; 67 \%$ and $77 \% \mathrm{Cr} / \mathrm{Pb}$; and $90 \%$ and $92 \% \mathrm{Co} / \mathrm{Cu}$ at $\pm 1 \sigma$ of background while the $\mathrm{N}$ and $\mathrm{W}$ sectors ratios were not within the $95 \% \mathrm{CI}$ predicted models. Findings are analogous with Font et al. [28] who recognized patterns with specific metal ratios (e.g., $\mathrm{Cd} / \mathrm{Pb}, \mathrm{Cr} / \mathrm{Pb}, \mathrm{Cu} / \mathrm{Pb}$, $\mathrm{Cd} / \mathrm{Cu}$ ) downwind from industrial and population centers. Other studies also identified similar trends with respect to $\mathrm{Cu} / \mathrm{Pb}, \mathrm{Cr} / \mathrm{Pb}$, and $\mathrm{Co} / \mathrm{Cu}$ downwind from sources [41]. The $\mathrm{N}$ and $\mathrm{W}$ sectors for instance, showed ratios nearer to background areas; a dissimilar distribution compared with the $S$ and E sectors whose ratios exceeded background. Metal ratios (i.e., $\mathrm{Cu} / \mathrm{Pb}, \mathrm{Cr} / \mathrm{Pb}$, and $\mathrm{Co} / \mathrm{Cu}$ ) may be indicative of pollution entering the valley, mixing with local sources and exiting through the E sector.

It is well documented that vehicle emissions are a source of $\mathrm{NO}_{3}{ }^{-}$for instance, Fenn et al. [42] documented that localized sources of $\mathrm{NO}_{3}{ }^{-}$have minimal effect on indigenous flora when sparse. Their study further showed that elevated vehicle pollution in an area with sparse flora was likely the result of a wider problem; i.e., nearby population centers. Findings show $\mathrm{NO}_{3}{ }^{-}$is lower in lichen biomass located in the $\mathrm{S}$ and E sectors while higher in the $\mathrm{N}$ and $\mathrm{W}$ sectors. It is possible that the $\mathrm{S}$ and E sectors with elevated $\mathrm{Cu}$, perhaps, is a contributing factor to lower $\mathrm{NO}_{3}{ }^{-}$in lichen biomass. Authors Kumar et al. [43] recognized that lower $\mathrm{NO}_{3}{ }^{-}$is usually found in areas with elevated $\mathrm{Cu}$ as lichen is part algal, $\mathrm{Cu}$ will inhibit its growth rate [29]. They also documented that other toxic metals (i.e., Cd, 
Cr) will affect a variety of algal photosynthetic functions; impeding its viability for nitrogen fixation, energy transfer, and ultimately its development. Copper and $\mathrm{Cd}$, among other metals, are significantly higher in the $\mathrm{S}$ and $\mathrm{E}$ sectors and are likely the cause of $\mathrm{NO}_{3}{ }^{-}$being lowest in these areas.

\section{Conclusions}

This study was the first to evaluate metals in lichen biomass within the Las Vegas Valley. Lichens contain higher metals in the $\mathrm{S}$ and $\mathrm{E}$ sectors with $\mathrm{Al}, \mathrm{Cd}, \mathrm{Cu}, \mathrm{Cr}, \mathrm{Ni}, \mathrm{Pb}, \mathrm{V}$ and $\mathrm{Zn}$ likely anthropogenically enhanced. Trace metal and $\mathrm{NO}_{3}{ }^{-}$data correlates with PCA, DFA and windrose data. Also, $\mathrm{NO}_{3}{ }^{-}$patterns in the $\mathrm{E}$ and $\mathrm{S}$ sectors along with in the $\mathrm{N}$ and $\mathrm{W}$ sectors could be the result of metal deposition for instance, $\mathrm{NO}_{3}{ }^{-}$shows an inverse relationship when compared to metals; elevated $\mathrm{NO}_{3}{ }^{-}$where metals are low $(\mathrm{N}, \mathrm{W})$. Although the higher ratios identified in the $\mathrm{E}$ sector were expected based on wind patterns, elevated metals and low $\mathrm{NO}_{3}{ }^{-}$in the $\mathrm{S}$ sector were not anticipated.

Background ratios for $\mathrm{NO}_{3}{ }^{-}$were calculated from the loci outside the Valley and compared with sample loci within the Valley. Data show that metal and $\mathrm{NO}_{3}{ }^{-}$ratios across the Las Vegas Valley clustered into two identified areas, $\mathrm{E}-\mathrm{S}$ (high metals, low $\mathrm{NO}_{3}{ }^{-}$) and $\mathrm{N}-\mathrm{W}$ (low metals, high $\mathrm{NO}_{3}{ }^{-}$) sectors, suggestive of enhancement in the $\mathrm{S}$ and $\mathrm{W}$ sectors. Pollutants generated within the valley, and perhaps an influx from other population centers, are moving from the $\mathrm{S}$ sector to the north-northeast across the valley, exiting on the north and south side of Frenchman Mountain located in the E sector. Pollutants within the valley will likely continue to increase with population but possibly from sources outside of the valley. Finally, additional research into pollution contained in precipitation (e.g., rain, snow) across the Las Vegas needs to be performed as it relates to lichen and air pollution

Acknowledgments: We would like to thanks the United States Department of Interior, Bureau of Land Management, Las Vegas Office for their corporation and permit during this research. We would also like to thank the students of the College of Southern Nevada, Department of Physical Sciences, for their efforts in the field, laboratory and data interpretation. Furthermore, we would like to thank the NASA-CoP Grant (NNX14Q94A) for providing funding to students at the College of Southern Nevada. I would like to thank Dragos G Zaharescu for his help and guidance with PCA and DFA analysis. Finally, we would like to thank the reviewers for their time and expertise during the review process.

Author Contributions: Douglas B. Sims, Amanda C. Hudson, Ji Hye Park, and Vernon Hodge initiated the overall research concept, design and research efforts. Heidi Porter and W. Geoffrey Spaulding evaluated and identified the lichen biomass. Douglas B Sims, Amanda C Hudson completed the initial manuscript, analyzed and interpreted measured data; supervised experiments, managed experimental work and performed measurements of trace metals and Nitrate.

Conflicts of Interest: The Authors declare no conflict of interest.

\section{References}

1. United States Agency for Toxic Substances and Disease Registry (USATSDR). Toxic Substances. 2016. Available online: http:/ / www.atsdr.cdc.gov/toxicsubstances.html (accessed on 25 February 2016).

2. Balabanova, B.; Stafilov, T.; Sajn, R.; Baeeva, K. Characterization of heavy metals in lichen species Hypogymnia physodes and Evernia prunastri due to biomonitoring of air pollution in the vicinity of copper mine. Int. J. Environ. Res. 2012, 6, 779-794. [CrossRef]

3. Clarke, L.W.; Jenerette, G.D.; Bain, D.J. Urban legacies and soil management affect the concentration and speciation of trace metals in Los Angeles community garden soils. Environ. Pollut. 2015, 197, 1-12. [CrossRef] [PubMed]

4. Bollen, J. The value of air pollution co-benefits of climate policies: Analysis with a global sector-trade CGE model called WorldScan. Technol. Forecast. Soc. Chang. 2015, 90, 178-191. [CrossRef]

5. Zelm, R.V.; Preiss, P.; Goethem, T.V.; Dingenen, R.V.; Huijbregts, M. Regionalized life cycle impact assessment of air pollution on the global scale: Damage to human health and vegetation. Atmos. Environ. 2016, 134, 129-137. [CrossRef]

6. Cheng, Z.; Luo, L.; Wang, S.; Wang, Y.; Sharma, S.; Shimadera, H.; Wang, X.; Bressi, M.; Miranda, R.M.; Jiang, J.; et al. Status and characteristics of ambient $\mathrm{PM}_{2.5}$ pollution in global megacities. Environ. Int. 2016, 89-90, 212-221. [CrossRef] [PubMed] 
7. Hasheminassab, S.; Daher, N.; Ostro, B.D.; Sioutas, C. Long-term source apportionment of ambient fine particulate matter $\left(\mathrm{PM}_{2.5}\right)$ in the Los Angeles Basin: A focus on emissions reduction from vehicular sources. Environ. Pollut. 2014, 193, 54-64. [CrossRef] [PubMed]

8. Saffari, A.; Daher, N.; Shafer, M.M.; Schauer, J.J.; Sioutas, C. Seasonal and spatial variation of trace elements and metals in quasi-ultrafine $\left(\mathrm{PM}_{0.25}\right)$ particles in the Los Angeles metropolitan area and characterization of their sources. Environ. Pollut. 2013, 181, 14-23. [CrossRef] [PubMed]

9. Folkesson, L.; Andersson-Bringmark, E. Impoverishment of vegetation in a coniferous forest polluted by copper and zinc. Can. J. Bot. 1988, 66, 417-428. [CrossRef]

10. Dobben, H.F.; Wolterbeek, H.T.; Wamelink, G.W.; Ter Braak, C.J. Relationship between epiphytic lichens, trace elements and gaseous atmospheric pollutants. Environ. Pollut. 2001, 112, 163-169. [CrossRef]

11. Giordani, P. In the diversity of epiphytic lichens a reliable indicator of air pollution? A case study from Italy. Environ. Pollut. 2007, 146, 317-323. [CrossRef] [PubMed]

12. Uluozlu, O.D.; Kinalioglu, K.; Tuzen, M.; Soylak, M. Trace metal levels in lichen samples from roadsides in east Black Sea region, Turkey. Biomed. Environ. Sci. 2007, 20, 203-207. [CrossRef] [PubMed]

13. Cleavitt, N.L.; Hinds, J.W.; Poirot, R.L.; Geiser, L.H.; Dibble, A.C.; Leon, B.; Perron, R.; Pardo, L.H. Epiphytic macrolichen communities correspond to patterns of sulfur and nitrogen deposition in the northeastern United States. Bryologist 2015, 118, 304-324. [CrossRef]

14. McMurray, J.A.; Roberts, D.W. Using Epiphytic lichens to monitor nitrogen deposition near natural gas drilling operations in the Wind River Range, WY, USA. Water Air Soil Pollut. 2013, 224, 1-14. [CrossRef]

15. Giordano, S.; Adamo, P.; Sorbo, S.; Vingiani, S. Atmospheric trace metal pollution in the Naples urban area based on results from moss and lichen bags. Environ. Pollut. 2005, 136, 431-442. [CrossRef] [PubMed]

16. Jeran, Z.; Jacimovic, R.; Batic, F.; Mavsar, R. Lichens as integrating air pollution monitors. Environ. Pollut. 2002, 120, 107-113. [CrossRef]

17. Donovan, G.H.; Jovan, S.E.; Gatziolis, D.; Burstyn, I.; Michael, Y.L.; Amacher, M.C.; Monleon, V.J. Using an epiphytic moss to identify previously unknown sources of atmospheric cadmium pollution. Sci. Total Environ. 2016, 559, 84-93. [CrossRef] [PubMed]

18. Geiser, L.H.; Jovan, S.E.; Glavich, D.A.; Porter, M.K. Lichen-based critical loads for atmospheric nitrogen deposition in Western Oregon and Washington Forests, USA. Environ. Pollut. 2010, 158, 2412-2421. [CrossRef] [PubMed]

19. Proctor, M.C.F. The Growth Curve of the Crustose Lichen Buellia canescens (Dicks.) De Not. Neww Phytol. 1977, 79, 659-663. [CrossRef]

20. Wetmore, C.M. Lichens and Air Quality in Boundary Water Canoe Are of Superior National Forest. 1987. Available online: http:/ / gis.nacse.org/lichenair/doc/BWCA1987.pdf (accessed on 2 January 2017).

21. Atwater, T. Implications of plate tectonics for the Cenozoic tectonic evolution of western North America. Geol. Soc. Am. Bull. 1970, 81, 3513-3536. [CrossRef]

22. Besser, J.M.; Finger, S.E.; Church, S.E. Impacts of historical mining on aquatic ecosystems-An ecological risk assessment. In Integrated Investigations of Environmental Effects of Historical Mining in the Animas River Watershed San Juan County, Colorado; Church, S.E., von Guerard, P., Finger, S.E., Chap, D., Eds.; Professional Paper; USGS, Department of the Interior: Washington, DC, USA, 2008; Volume 1651, pp. 87-106. Available online: http:/ / pubs.usgs.gov/pp/1651/downloads/Vol1_combinedChapters/vol1_chapD.pdf (accessed on 15 August 2017).

23. Forrester, S.W. Provenance of the Miocene-Pliocene Muddy Creek Formation Near Mesquite, Nevada. Master's Thesis, University of Nevada, Las Vegas, NV, USA, 2009.

24. Longwell, C.R.; Pampeyan, E.H.; Bowyer, B.; Roberts, R.J. Geology and Mineral Deposits of Clark County, Nevada; Nevada Bureau of Mines and Geology: Reno, NV, USA, 1965.

25. Birks, H.J.B.; Birks, H.H. Quaternary Palaeoecology; University Park: Baltimore, MD, USA, 1980; p. 289.

26. USEPA. Test Methods for Evaluating Solids and Wastes_Physical/Chemical Methods (SW-846); U.S. Environmental Protection Agency: Washington, DC, USA, 1997.

27. Boltersdorf, S.H.; Pesch, R.; Werner, W. Comparative use of lichens, mosses and tree bark to evaluate nitrogen deposition in Germany. Environ. Pollut. 2014, 189, 43-53. [CrossRef] [PubMed]

28. Font, A.; Hoogh, K.D.; Leal-Sanchez, M.; Ashworth, D.C.; Brown, R.J.C.; Hansell, A.L.; Fuller, G.W. Using metal ratios to detect emissions from municipal waste incinerators in ambient air pollution data. Atmos. Environ. 2015, 113, 177-186. [CrossRef] 
29. Hauck, M.; Boning, J.; Jacob, M.; Dittrich, S.; Feussner, I.; Leuschner, C. Lichen substance concentrations in lichen Hypogymnia physodes are correlated with heavy metal concentrations in the substratum. Environ. Exp. Bot. 2013, 85, 58-63. [CrossRef]

30. Botsou, F.; Sungur, A.; Kelepertzid, E.; Soylak, M. Insights into the chemical partitioning of trace metals in roadside and off-road agricultural soils along two major highways in Attica's region, Greece. Ecotox. Environ. Safe. 2016, 132, 101-110. [CrossRef] [PubMed]

31. Liu, H.; Chen, L.P.; Ai, Y.; Yang, X.; Yu, Y.; Zuo, Y.; Fu, G. Heavy metal contamination in soil alongside mountain railway in Sichuan, China. Environ. Monit. Assess. 2009, 152, 25-33. [CrossRef] [PubMed]

32. Zhang, H.; Wang, Z.; Zhang, Y.; Hu, Z. The effects of the Qinghai-Tibet railway on heavy metals enrichment in soils. Sci. Total Environ. 2012, 439, 240-248. [CrossRef] [PubMed]

33. AIRNow. Clark County-Department of Air Quality Management (CCDAQM), Nevada Division of Environmental Protection-Bureau of Air Quality Planning, Washoe County Health District-Air Quality Management Division. 2017. Available online: https:/ / airnow.gov/index.cfm?action=airnow.local_city\& cityid=120 (accessed on 7 July 2017).

34. Delamater, P.L.; Finley, A.O.; Banerjee, S. An analysis of asthma hospitalizations, air pollution, and weather conditions in Los Angeles County, California. Sci. Total Environ. 2012, 425, 110-118. [CrossRef] [PubMed]

35. Loppi, S.; Frati, L.; Paoli, L.; Bigagli, V.; Rossetti, C.; Bruscoli, C.; Corsini, A. Biodiversity of epiphytic lichens and heavy metal contents of Flavoparmelia caperata thalli as indicators of temporal variations of air pollution in the town of Montecatini Terme (central Italy). Sci. Total Environ. 2004, 326, 113-122. [CrossRef] [PubMed]

36. Purvis, O.W. Interactions of lichens with metals. Sci. Prog. 1996, 79, 283-309.

37. Spribille, T.; Tuovinen, V.; Resl, P.; Vanderpool, D.; Wolinski, H.; Aime, M.C.; Schneider, K.; Stabentheiner, E.; Toome-Heller, M.; Thor, G.; et al. Basidiomycete yeasts in the cortex of ascomycete macrolichens. Science 2016, 353, 488-492. [CrossRef] [PubMed]

38. Lodenius, M.; Kiiskinen, J.; Tulisalo, E. Metal levels in an epiphytic lichen as indicators of air quality in a suburb of Helsinki, Finland. Boreal Environ. Res. 2010, 15, 446-452. [CrossRef]

39. Demiray, A.D.; Yolcubal, I.; Akyol, N.H.; Cobanoglu, G. Biomonitoring of airborne metals using the Lichen Xanthoria parietina in Kocaeli Province, Turkey. Ecol. Indic. 2012, 18, 632-643. [CrossRef]

40. Ungureanu, T.; Iancu, G.O.; Pintilei, M.; Chicos, M.M. Spatial distribution and geochemistry of heavy metals in soils: A case study from the NE area of Vaslui County, Romania. J. Geochem. Explor. 2017, 176, 20-32. [CrossRef]

41. Pacyna, E.G.; Pacyna, J.M.; Fudala, J.; Strzelecka-Jastrzab, E.; Hlawiczka, S.; Panasiuk, D.; Nitter, S.; Pregger, T.; Pfeiffer, H.; Friedrich, R. Current and future emissions of selected heavy metals to the atmosphere from anthropogenic sources in Europe. Atmos. Environ. 2007, 41, 8557-8566. [CrossRef]

42. Fenn, M.E.; Geiser, L.; Bachman, R.; Blubaugh, T.J.; Bytnerowicz, A. Atmospheric deposition inputs and effects on lichen chemistry and indicator species in the Columbia River Gorge, USA. Environ. Pollut. 2007, 146, 77-91. [CrossRef] [PubMed]

43. Kumar, K.S.; Han, Y.; Choo, K.; Kong, J.; Han, T. Chlorophyll fluorescence based copper toxicity assessment of two algal species. Toxicol. Environ. Health Sci. 2009, 1, 17-23. [CrossRef]

(C) 2017 by the authors. Licensee MDPI, Basel, Switzerland. This article is an open access article distributed under the terms and conditions of the Creative Commons Attribution (CC BY) license (http:// creativecommons.org/licenses/by/4.0/). 